\title{
The noise of cluster mass reconstructions from a source redshift distribution
}

\author{
M. Lombardi, P. Schneider, and C. Morales-Merino \\ Institüt für Astrophysik und Extraterrestrische Forschung, Auf dem Hügel 71, 53121 Bonn, Germany
}

Received 15 May 2001 / Accepted 13 November 2001

\begin{abstract}
The parameter-free reconstruction of the surface-mass density of clusters of galaxies is one of the principal applications of weak gravitational lensing. From the observable ellipticities of images of background galaxies, the tidal gravitational field (shear) of the mass distribution is estimated, and the corresponding surface mass density is constructed. The noise of the resulting mass map is investigated here, generalizing previous work which included mainly the noise due to the intrinsic galaxy ellipticities. Whereas this dominates the noise budget if the lens is very weak, other sources of noise become important, or even dominant, for the medium-strong lensing regime close to the center of clusters. In particular, shot noise due to a Poisson distribution of galaxy images, and increased shot noise owing to the correlation of galaxies in angular position and redshift, can yield significantly larger levels of noise than that from the intrinsic ellipticities only. We estimate the contributions from these various effects for two widely used smoothing operations, showing that one of them effectively removes the Poisson and the correlation noises related to angular positions of galaxies. Noise sources due to the spread in redshift of galaxies are still present in the optimized estimator and are shown to be relevant in many cases. We show how (even approximate) redshift information can be profitably used to reduce the noise in the mass map. The dependence of the various noise terms on the relevant parameters (lens redshift, strength, smoothing length, redshift distribution of background galaxies) are explicitly calculated and simple estimates are provided.
\end{abstract}

Key words. cosmology: dark matter - gravitational lensing - large-scale structure of universe - galaxies: clusters: general - methods: statistical

\section{Introduction}

Weak gravitational lensing has been recognized as a powerful tool to investigate the mass and mass distribution of clusters of galaxies (Webster 1985; Tyson et al. 1990; Kochanek 1990). In their pioneering paper Kaiser \& Squires (1993) pointed out that the tidal gravitational field of the cluster, as measured from the distortion of image shapes of the faint background galaxy population, can be used to reconstruct the two-dimensional projected mass profiles of clusters, without referring to a parameterized mass model. This method, modified in various ways later (see, e.g., Kaiser 1995; Schneider 1995; Schneider \& Seitz 1995; Bartelmann et al. 1996; Squires \& Kaiser 1996; Seitz \& Schneider 1997; Seitz et al. 1998; Lombardi \& Bertin 1998a,b; Bartelmann \& Schneider 2001 for a recent review) has been applied to more than a dozen clusters up to now (e.g. Fahlman et al. 1994; Smail et al. 1995; Squires et al. 1996; Squires et al. 1996; Seitz et al. 1996; Luppino \& Kaiser 1997; Clowe et al. 1998; Hoekstra et al. 1998;

Send offprint requests to: M. Lombardi, e-mail: lombardi@astro.uni-bonn.de
Mellier 1999). One of the main results from these studies is that in many clusters the projected mass distribution closely follows the distribution of luminous cluster galaxies.

Recently, a number of papers have been devoted to the study of the noise properties of weak lensing mass reconstructions. Such studies are important to optimize the lensing techniques used. For example, Lombardi \& Bertin (1998a) have provided estimates for the noise properties of weak lensing mass maps, showing also that a particular technique, previously described by Seitz \& Schneider (1996), leads to optimal maps in the linear regime; analogously, van Waerbeke (2000) has extended these results to maximum likelihood estimators.

All these analytical studies, however, have been carried out using a "mean field" approximation, thus ignoring some contributions to the noise. In particular, Poisson noise and correlations on the positions of source galaxies are not taken into account. In this paper we consider in detail the effects of these sources of noise and we provide analytical estimates for them. We also show that, in many situations, these extra contributions to the noise are 
non-negligible. In particular, as already pointed out by Bertin \& Lombardi (2001), the noise due to correlations of the source galaxies does not decrease with the density of observed galaxies, and thus puts an intrinsic limit on the accuracy of weak lensing mass maps. On the other hand, we also show that the use of some redshift information can significantly reduce the noise due to the correlation of galaxies and, at least partially, the Poisson noise.

The paper is organized as follows. We briefly describe standard weak lensing mass reconstruction techniques in Sect. 2. In Sect. 3 we introduce the statistical methods used to study the properties of the shear and the mass maps obtained in weak lensing reconstructions, and we apply those methods to obtain some general results. A very useful approximation technique is discussed in Sect. 4. This technique allows us to obtain simple analytical results and to study the impact of various sources of noise on the shear estimation. A more accurate shear estimator, which leads to significantly smaller noise, is studied in Sect. 5. Section 7 is devoted to the impact of observations on reconstructions of weak lenses. If information on the source redshifts is available, then some sources of noise can be significantly reduced. Two different estimators that can take advantage of redshift information are discussed in Sect. 8. Finally, the main results obtained in this paper are summarized in Sect. 9. In order to simplify the discussion and to avoid long calculations, we have moved several derivations to the appendices. In particular, in Appendix A we recall a standard statistical technique; in Appendices B and C we carry out the statistical analysis for the estimator of Sect. 5; in Appendix D we sketch the calculations for the estimators of Sect. 8.

The weak lensing approximation is taken to be valid in this paper, so that the shear is taken as an observable (see below Eq. (1)). The Einstein-de Sitter cosmological model is assumed and the Hubble constant is parameterized as $H_{0}=100 h \mathrm{~km} \mathrm{~s}^{-1} \mathrm{Mpc}^{-1}$.

\section{Local shear estimators}

In the weak lensing limit, the observed ellipticity $\epsilon^{(n)}$ of a galaxy $(n \in\{1,2, \ldots, N\}$ denotes the galaxy considered) is related to the true source ellipticity $\epsilon^{\mathrm{s}(n)}$ :

$\epsilon^{(n)}=\epsilon^{\mathrm{s}(n)}+Z^{(n)} \gamma\left(\boldsymbol{\theta}^{(n)}\right)$,

where $\gamma(\boldsymbol{\theta})$ is the lens shear field for fiducial sources at infinite redshift, $Z^{(n)}=Z\left(z^{(n)}\right)$ is the cosmological weight for the $n$-th galaxy, and $\boldsymbol{\theta}^{(n)}$ is its observed position on the sky. We recall that $Z(z)$ depends on the assumed cosmological model, on the redshift $z_{\mathrm{d}}$ of the lens, and on the redshift $z^{(n)}$ of the galaxy. More precisely, $Z(z)$ can be written as a ratio of angular diameter distances between the observer and the lens, the observer and the source, and the lens and the source (see, e.g., Bartelmann \& Schneider 2001).

Equation (1) provides a simple way to infer the shear of a lens. Since source galaxies can be taken to be randomly oriented, so that $\left\langle\epsilon^{\mathrm{s}}\right\rangle=0$, the average of galaxies close on angular position is a good estimator for the shear. The average, needed in order to obtain a high signal-to-noise estimator from extremely noisy data (the source ellipticity), is usually performed using a function $W\left(\boldsymbol{\theta}-\boldsymbol{\theta}^{\prime}\right)$ which describes the relative contribution of a galaxy located at $\boldsymbol{\theta}^{\prime}$ to the shear at the position $\boldsymbol{\theta}$. Since only relative values of $W$ are important, we can assume that this function is normalized,

$\int_{\Omega} W\left(\boldsymbol{\theta}-\boldsymbol{\theta}^{\prime}\right) \mathrm{d}^{2} \theta^{\prime}=1$,

where $\Omega$ is the field in the sky where the observations are made. In principle, the normalization condition written above cannot be satisfied for all points $\boldsymbol{\theta}$ of $\Omega$ unless $W$ is constant. In practice, if the area of observation $\Omega$ is large compared to the scale of $W$, we can assume that Eq. (2) holds for nearly all points $\boldsymbol{\theta} \in \Omega$, being false only for the points close to the boundary of the field. This approximation, called framing (see Lombardi \& Bertin 1998b), greatly simplifies the analysis. In the following, we will always use this approximation. Often, in weak lensing studies, a Gaussian weight function is used:

$W\left(\boldsymbol{\theta}-\boldsymbol{\theta}^{\prime}\right)=\frac{1}{2 \pi \sigma_{W}^{2}} \exp \left(-\frac{\left|\boldsymbol{\theta}-\boldsymbol{\theta}^{\prime}\right|^{2}}{2 \sigma_{W}^{2}}\right)$.

Using the smoothing function $W$, two simple unbiased estimators for the shear can be provided. The first one is the traditional Kaiser \& Squires (1993) estimates for the shear:

$\hat{\gamma}(\boldsymbol{\theta})=\frac{1}{\langle Z\rangle \rho} \sum_{n=1}^{N} \epsilon^{(n)} W\left(\boldsymbol{\theta}-\boldsymbol{\theta}^{(n)}\right)$.

Here, $\rho$ is the density of observed galaxies, for simplicity taken to be constant on the whole field of observation (as a reference value, a density of 30 galaxies per square arcmin can be easily reached today). In this equation and in the whole paper we will use the hat $\left(^{\wedge}\right)$ to denote estimated or measured quantities. Equation (4) is very simple, but has the disadvantage of being significantly affected by Poisson noise. In fact, it is reasonable to expect some variations of the local density of galaxies in the field. In such cases, we would have additional noise on $\gamma$ simply due to changes on $\rho$.

As argued by Seitz \& Schneider (1995), since the positions of galaxies are readily available, we can modify the estimator into

$\hat{\gamma}(\boldsymbol{\theta})=\frac{\sum_{n=1}^{N} \epsilon^{(n)} W\left(\boldsymbol{\theta}-\boldsymbol{\theta}^{(n)}\right)}{\langle Z\rangle \sum_{n=1}^{N} W\left(\boldsymbol{\theta}-\boldsymbol{\theta}^{(n)}\right)}$.

We will refer to this estimator as balanced, because it, in contrast to (4), explicitely takes into account the local number density of source galaxies. Equation (5) is actually often used in weak lensing studies (see, e.g., Lombardi et al. 2000).

In this paper we will study in detail both estimators and obtain their expected variances. We will consider initially the estimator (4), which is much easier to study 
than (5). Although non-optimal, this estimator is very important for many reasons: (i) It is the first one considered in the literature, in the classical paper by Kaiser \& Squires (1993); (ii) it is simpler to study and hence allows exact analytical results; (iii) it has been used in the literature (e.g. Fahlman et al. 1994; Tyson \& Fischer 1995) and actually is still used by some authors (Fischer et al. 1997; Fischer \& Tyson 1997; Luppino \& Kaiser 1997; Fischer 1999). We will thus delay the study of Eq. (5) till Sect. 5, hoping also that a detailed analysis of the unbalanced estimator will make the complex discussion of the balanced one easier to follow and results interpreted.

An estimate of the shear can be used to obtain an estimate for the projected, dimensionless mass distribution $\kappa(\boldsymbol{\theta})$ of the lens using well-know techniques (see, e.g., Seitz \& Schneider 1996; Lombardi \& Bertin 1998a, 1999a). In general, because of the linear relationship between $\gamma$ and $\kappa$, we can write

$\kappa(\boldsymbol{\theta})=\bar{\kappa}+\int_{\Omega} \mathcal{D}_{i}\left(\boldsymbol{\theta}, \boldsymbol{\theta}^{\prime}\right) \gamma_{i}\left(\boldsymbol{\theta}^{\prime}\right) \mathrm{d}^{2} \theta^{\prime}$,

where the Einstein's convention on repeated indeces has been used. In this equation, $\bar{\kappa}$ is the mean value of $\kappa$ on $\Omega$ and $\mathcal{D}_{i}\left(\boldsymbol{\theta}, \boldsymbol{\theta}^{\prime}\right)$ is a suitable kernel. For example, in the limit where the area of observation $\Omega$ is much larger than the size of the lensing cluster, a simple kernel can be used (Kaiser \& Squires 1993):

$$
\left(\begin{array}{c}
\mathcal{D}_{1}^{\mathrm{KS}} \\
\mathcal{D}_{2}^{\mathrm{KS}}
\end{array}\right)(\boldsymbol{\theta}, \boldsymbol{\theta}+\boldsymbol{\phi})=\frac{1}{\pi|\boldsymbol{\phi}|^{4}}\left(\begin{array}{c}
\phi_{1}^{2}-\phi_{2}^{2} \\
2 \phi_{1} \phi_{2}
\end{array}\right) .
$$

Note that in this case Eq. (6) is a simple convolution. For finite fields $\Omega$, the kernel $\mathcal{D}_{i}\left(\boldsymbol{\theta}, \boldsymbol{\theta}^{\prime}\right)$ often cannot be written in closed form, still it can be written in terms of the Green function for a Neumann problem (see Lombardi \& Bertin 1998a; Seitz \& Schneider 2001). Fast numerical methods exist to obtain $\kappa$ from the shear $\gamma$ (Lombardi \& Bertin 1999a).

The linear relationship between $\kappa$ and $\gamma$ allows us to easily derive the statistical properties of the mass map from the properties of the shear. In the following, thus, we will first evaluate the expectation value and the noise of the shear, and then obtain analogous results for the mass map.

\section{Statistical analysis}

In this section we will perform a statistical analysis of the shear estimator (4) using a general probability density distribution for all random variables involved. As already noted above, this estimator is not optimal and generally should not be used. One purpose of this paper is actually to show that the increase in noise of unbalanced estimator is very large in most cases.

Let us describe in detail the various random variables used.

Source ellipticities $\epsilon^{\mathrm{s}(n)}$. We assume that the intrinsic ellipticity of each galaxy follows the probability distribution $p_{\epsilon}\left(\epsilon^{\mathrm{s}}\right)$; this probability distribution is taken to be isotropic, so that $\left\langle\epsilon^{\mathrm{s}(n)}\right\rangle=0$. Moreover, we assume no correlation between ellipticities of different galaxies, and no correlation between ellipticities and other random variables.

Source redshifts $z^{(n)}$. The redshifts of sources are taken to be unknown in the first part of this paper (see Sect. 8 for the case where some knowledge on redshifts is available). We assume that each redshift follows the probability distribution $p_{z}(z)$. Correlations in redshifts and between redshifts and positions are considered.

Observed position $\boldsymbol{\theta}^{(n)}$. The positions of galaxies are obviously readily available in weak lensing observations. However, in order to obtain general results, we will perform an ensemble averaging on galaxy positions. This way, we will obtain results which are not strictly dependent on the particular configuration of galaxies. Here we will assume that observed positions follow a uniform distribution on the field of observation $\Omega$ (thus neglecting the magnification bias). Correlations in positions and between positions and redshifts are considered.

In summary, the average of a function $f$ of random variables of a single galaxy will be performed using the relation

$$
\begin{array}{r}
\left\langle f\left(\epsilon^{(n)}, z^{(n)}, \boldsymbol{\theta}^{(n)}\right)\right\rangle=\frac{1}{A} \int_{\Omega} \mathrm{d}^{2} \theta \int_{0}^{\infty} p_{z}(z) \mathrm{d} z \\
\times \int_{\left|\epsilon^{\mathrm{s}}\right|<1} p_{\epsilon}\left(\epsilon^{\mathrm{s}}\right) f\left(\epsilon^{\mathrm{s}}+Z(z) \gamma(\boldsymbol{\theta}), z, \boldsymbol{\theta}\right) \mathrm{d}^{2} \epsilon^{\mathrm{s}} .
\end{array}
$$

Here $A$ is the area of the observation set $\Omega$. Similarly, the average of the product of two functions $f$ and $g$ of random variables of two different galaxies $(n \neq m)$ will be performed using the relation

$$
\begin{aligned}
& \left\langle f\left(\epsilon^{(n)}, z^{(n)}, \boldsymbol{\theta}^{(n)}\right) g\left(\epsilon^{(m)}, z^{(m)}, \boldsymbol{\theta}^{(m)}\right)\right\rangle= \\
& \quad\left\langle f\left(\epsilon^{(n)}, z^{(n)}, \boldsymbol{\theta}^{(n)}\right)\right\rangle \cdot\left\langle g\left(\epsilon^{(m)}, z^{(m)}, \boldsymbol{\theta}^{(m)}\right)\right\rangle \\
& \quad+\frac{1}{A^{2}} \int_{\Omega} \mathrm{d}^{2} \theta \int_{\Omega} \mathrm{d}^{2} \theta^{\prime} \int_{0}^{\infty} p_{z}(z) \mathrm{d} z \int_{0}^{\infty} p_{z}\left(z^{\prime}\right) \mathrm{d} z^{\prime} \\
& \quad \times \int_{\left|\epsilon^{\mathrm{s}}\right|<1} p_{\epsilon}\left(\epsilon^{\mathrm{s}}\right) \mathrm{d}^{2} \epsilon^{\mathrm{s}} \int_{\left|\epsilon^{\mathrm{s}}\right|<1} p_{\epsilon}\left(\epsilon^{\mathrm{s}}\right) f(\epsilon, z, \boldsymbol{\theta}) g\left(\epsilon^{\prime}, z^{\prime}, \boldsymbol{\theta}^{\prime}\right) \xi \mathrm{d}^{2} \epsilon^{\mathrm{s} \prime} .
\end{aligned}
$$

In this relation, for simplicity, we have kept the original arguments $\epsilon=\epsilon^{\mathrm{s}}+Z(z) \gamma(\boldsymbol{\theta})$ and $\epsilon^{\prime}=\epsilon^{\mathrm{s} \prime}+Z\left(z^{\prime}\right) \gamma\left(\boldsymbol{\theta}^{\prime}\right)$ for $f$ and $g$ in the last term. The function $\xi$, for our purposes, can be written as

$\xi=\xi\left(\boldsymbol{\theta}-\boldsymbol{\theta}^{\prime}, z, z^{\prime}\right)$

The galaxy two-point correlation function is often written in terms of the proper distance $d$ between the galaxies. Observations show that the local galaxy two-point correlation function is very well approximated in the range $10 \mathrm{kpc}<h d<10 \mathrm{Mpc}$ by the power law

$\xi(d)=\left(\frac{d_{0}}{d}\right)^{\eta}$ 
with $d_{0} \simeq(5.4 \pm 1) h^{-1} \mathrm{Mpc}$, and $\eta \simeq 1.77 \pm 0.04$ (Peebles 1993). At high redshift $(z \sim 1)$, the two-point correlation function is more poorly known. The redshift-dependence is often written as a power-law

$\xi(d, z)=\left(\frac{d_{0}}{d}\right)^{\eta}(1+z)^{\alpha}$.

Recent investigations (Le Fèvre et al. 1996) suggest that $\alpha$ is in the range $[-3,-1]$. In this paper, then, we will take the fiducial value $\alpha=-2$.

Finally, we note that, given the linear relationship between $\epsilon$ and $\epsilon^{\mathrm{s}}$, it is not difficult to include the effect of errors of measurements for the observed ellipticities in our discussion. In general, such errors can be described using a Baysian probability distribution $p_{\text {obs }}(\hat{\epsilon} \mid \epsilon)$ which gives the probability of measuring $\hat{\epsilon}$ when the real lensed ellipticity is $\epsilon$. Then the standard framework described above can be applied without any change if we replace the probability distribution $p\left(\epsilon^{\mathrm{s}}\right)$ with an effective distribution $p_{\text {eff }}\left(\epsilon^{\mathrm{s}}\right)$ given by

$p_{\text {eff }}\left(\hat{\epsilon}^{\mathrm{S}}\right)=\int_{\left|\epsilon^{\mathrm{s}}\right|<1} p_{\text {obs }}\left(\hat{\epsilon}^{\mathrm{S}} \mid \epsilon^{\mathrm{S}}\right) p_{\epsilon}\left(\epsilon^{\mathrm{s}}\right) \mathrm{d}^{2} \epsilon^{\mathrm{s}}$.

We stress that, because of the linearity of Eq. (1), the distribution $p_{\text {obs }}\left(\hat{\epsilon}^{\mathrm{S}} \mid \epsilon^{\mathrm{S}}\right)$ is formally identical to the distribution $p_{\text {obs }}(\hat{\epsilon} \mid \epsilon)$. Finally, we observe that if this Baysian distribution is taken to depend only on the difference $|\hat{\epsilon}-\epsilon|$, then the effective variance $\sigma_{\epsilon \mathrm{eff}}^{2}$ of $\epsilon^{\mathrm{s}}$ can be written as

$\sigma_{\text {eff }}^{2}=\sigma_{\epsilon}^{2}+\sigma_{\text {obs }}^{2}$,

where $\sigma_{\text {obs }}^{2}$ is the variance of $(\hat{\epsilon}-\epsilon)$ (given by $p_{\text {obs }}$ ), and $\sigma_{\epsilon}^{2}$ is the variance of the source ellipticities, defined as $\left\langle\epsilon_{i}^{\mathrm{s}} \epsilon_{j}^{\mathrm{s}}\right\rangle=\sigma_{\epsilon}^{2} \delta_{i j}$.

\subsection{Average value}

The average value of the estimator (4) is easily evaluated using Eq. (8):

$\langle\hat{\gamma}(\boldsymbol{\theta})\rangle=\int_{\Omega} W\left(\boldsymbol{\theta}-\boldsymbol{\theta}^{\prime}\right) \gamma\left(\boldsymbol{\theta}^{\prime}\right) \mathrm{d}^{2} \theta^{\prime}$.

Because of the linearity between $\gamma$ and $\kappa$ we also obtain

$\langle\hat{\kappa}(\boldsymbol{\theta})\rangle=\int_{\Omega} W\left(\boldsymbol{\theta}-\boldsymbol{\theta}^{\prime}\right) \kappa\left(\boldsymbol{\theta}^{\prime}\right) \mathrm{d}^{2} \theta^{\prime}$.

In other words, the expected value of the estimated mass map is the true mass map smoothed by the weight function $W$. The length-scale of this function, thus, sets the resolution of the shear and mass map.

\subsection{Covariance}

A complete characterization of the error on the shear map is given by the two-point correlation function, defined as (see Lombardi \& Bertin 1998a)

$\operatorname{Cov}_{i j}\left(\hat{\gamma} ; \boldsymbol{\theta}, \boldsymbol{\theta}^{\prime}\right)=\left\langle\left[\hat{\gamma}_{i}(\boldsymbol{\theta})-\left\langle\hat{\gamma}_{i}(\boldsymbol{\theta})\right\rangle\right]\left[\hat{\gamma}_{j}\left(\boldsymbol{\theta}^{\prime}\right)-\left\langle\hat{\gamma}_{j}\left(\boldsymbol{\theta}^{\prime}\right)\right\rangle\right]\right\rangle$.
Analogously, we define the two-point correlation function for the mass map as

$\operatorname{Cov}\left(\hat{\kappa} ; \boldsymbol{\theta}, \boldsymbol{\theta}^{\prime}\right)=\left\langle[\hat{\kappa}(\boldsymbol{\theta})-\langle\hat{\kappa}(\boldsymbol{\theta})\rangle]\left[\hat{\kappa}\left(\boldsymbol{\theta}^{\prime}\right)-\left\langle\hat{\kappa}\left(\boldsymbol{\theta}^{\prime}\right)\right\rangle\right]\right\rangle \cdot$

Equation (6) provides a simple way to obtain $\operatorname{Cov}(\hat{\kappa})$ from $\operatorname{Cov}(\hat{\gamma})$ :

$$
\begin{aligned}
\operatorname{Cov}\left(\hat{\kappa} ; \boldsymbol{\theta}, \boldsymbol{\theta}^{\prime}\right)= & \int_{\Omega} \mathrm{d}^{2} \phi \mathcal{D}_{i}(\boldsymbol{\theta}, \boldsymbol{\phi}) \int_{\Omega} \mathrm{d}^{2} \phi^{\prime} \mathcal{D}_{j}\left(\boldsymbol{\theta}^{\prime}, \boldsymbol{\phi}^{\prime}\right) \\
& \times \operatorname{Cov}_{i j}\left(\hat{\gamma} ; \boldsymbol{\phi}, \boldsymbol{\phi}^{\prime}\right)
\end{aligned}
$$

We stress that the simple variance of $\hat{\gamma}$, i.e. the quantity $\operatorname{Cov}_{i i}(\hat{\gamma}, \boldsymbol{\theta}, \boldsymbol{\theta})$, would not be sufficient to evaluate the variance of $\hat{\kappa}$.

In order to obtain $\operatorname{Cov}(\hat{\gamma})$ we write the scatter of $\hat{\gamma}$ from its average value as

$$
\begin{aligned}
{\left[\hat{\gamma}_{i}-\left\langle\hat{\gamma}_{i}\right\rangle\right](\boldsymbol{\theta})=} & \frac{1}{\langle Z\rangle \rho} \sum_{n=1}^{N}\left[\left(\epsilon_{i}^{\mathrm{s}(n)}+\gamma_{i}\left(\boldsymbol{\theta}^{(n)}\right) Z^{(n)}\right) W\left(\boldsymbol{\theta}-\boldsymbol{\theta}^{(n)}\right)\right. \\
& \left.-\frac{\langle Z\rangle}{A} \int_{\Omega} W(\boldsymbol{\theta}-\boldsymbol{\phi}) \gamma_{i}(\boldsymbol{\phi}) \mathrm{d}^{2} \phi\right]
\end{aligned}
$$

since $N=A \rho$. Hence we find

$$
\begin{aligned}
& \operatorname{Cov}_{i j}\left(\hat{\gamma} ; \boldsymbol{\theta}, \boldsymbol{\theta}^{\prime}\right)= \\
& \frac{1}{\langle Z\rangle^{2} \rho^{2}}\left\langle\sum _ { n = 1 } ^ { N } \left[\left(\epsilon_{i}^{\mathrm{s}(n)}+\gamma_{i}\left(\boldsymbol{\theta}^{(n)}\right) Z^{(n)}\right) W\left(\boldsymbol{\theta}-\boldsymbol{\theta}^{(n)}\right)\right.\right.\left.-\frac{\langle Z\rangle}{A} \int_{\Omega} W(\boldsymbol{\theta}-\boldsymbol{\phi}) \gamma_{i}(\boldsymbol{\phi}) \mathrm{d}^{2} \phi\right] \\
& \times \sum_{m=1}^{N}\left[\left(\epsilon_{j}^{\mathrm{s}(m)}+\gamma_{j}\left(\boldsymbol{\theta}^{(m)}\right) Z^{(m)}\right) W\left(\boldsymbol{\theta}^{\prime}-\boldsymbol{\theta}^{(m)}\right)\right. \\
&\left.\left.\quad-\frac{\langle Z\rangle}{A} \int_{\Omega} W\left(\boldsymbol{\theta}^{\prime}-\boldsymbol{\phi}^{\prime}\right) \gamma_{j}\left(\boldsymbol{\phi}^{\prime}\right) \mathrm{d}^{2} \phi^{\prime}\right]\right\rangle
\end{aligned}
$$

This expression is evaluated more easily if we distinguish the two situations $n=m$ and $n \neq m$. Let us start with the case $n=m$.

If $n=m$, then we are considering the same galaxy and Eq. (8) must be applied. In this case, using the isotropy hypothesis for source ellipticities, we obtain

$$
\begin{aligned}
C_{1}= & \frac{\sigma_{\epsilon}^{2} \delta_{i j}}{\langle Z\rangle^{2} \rho} \int_{\Omega} W(\boldsymbol{\theta}-\boldsymbol{\phi}) W\left(\boldsymbol{\theta}^{\prime}-\boldsymbol{\phi}\right) \mathrm{d}^{2} \phi \\
& +\frac{\left\langle Z^{2}\right\rangle}{\langle Z\rangle^{2} \rho} \int_{\Omega} \gamma_{i}(\boldsymbol{\phi}) \gamma_{j}(\boldsymbol{\phi}) W(\boldsymbol{\theta}-\boldsymbol{\phi}) W\left(\boldsymbol{\theta}^{\prime}-\boldsymbol{\phi}\right) \mathrm{d}^{2} \phi \\
& -\frac{1}{\rho^{2} A^{2}} \int_{\Omega} W(\boldsymbol{\theta}-\boldsymbol{\phi}) \gamma_{i}(\boldsymbol{\phi}) \mathrm{d}^{2} \phi \\
& \times \int_{\Omega} W\left(\boldsymbol{\theta}^{\prime}-\boldsymbol{\phi}^{\prime}\right) \gamma_{j}\left(\boldsymbol{\phi}^{\prime}\right) \mathrm{d}^{2} \phi^{\prime}
\end{aligned}
$$

The last term vanishes in the limit where the areaof observation $A$ is large. [What actually matters here is the 
ratio between the "effective" area of the weight function $W$ and $A$.] We thus will ignore this term and similar terms arising in other expressions.

When $n \neq m$, on the other hand, we obtain a single term involving the two-point correlation function

$$
\begin{aligned}
C_{2}= & \frac{1}{\langle Z\rangle^{2}} \int_{\Omega} \mathrm{d}^{2} \phi W(\boldsymbol{\theta}-\boldsymbol{\phi}) \gamma_{i}(\boldsymbol{\phi}) \int_{\Omega} \mathrm{d}^{2} \phi^{\prime} W\left(\boldsymbol{\theta}^{\prime}-\boldsymbol{\phi}^{\prime}\right) \gamma_{j}\left(\boldsymbol{\phi}^{\prime}\right) \\
& \times \int_{0}^{\infty} p_{z}(z) Z(z) \mathrm{d} z \int_{0}^{\infty} p_{z}\left(z^{\prime}\right) Z\left(z^{\prime}\right) \xi\left(\boldsymbol{\phi}-\boldsymbol{\phi}^{\prime}, z, z^{\prime}\right) \mathrm{d} z^{\prime} .
\end{aligned}
$$

The last two integrals in the r.h.s. of this equation are reminiscent of Limber's equation (see Peebles 1993) and could suggest that the (projected) angular correlation function is relevant for lensing. This function, in fact, can be written in term of the full three-dimensional correlation $\xi$ as

$w(\boldsymbol{\varphi})=\int_{0}^{\infty} p_{z}(z) \mathrm{d} z \int_{0}^{\infty} p_{z}\left(z^{\prime}\right) \xi\left(\boldsymbol{\varphi}, z, z^{\prime}\right) \mathrm{d} z^{\prime}$.

In reality, because of the presence of the cosmological weights $Z(z)$ and $Z\left(z^{\prime}\right)$ in the integrand of Eq. (23), the expression for $C_{2}$ cannot be rewritten in terms of the angular correlation $w(\boldsymbol{\varphi})$ only.

To simplify the notation, we will write terms similar to the one found in Eq. (23) as

$C_{2}=\frac{1}{\langle Z\rangle^{2}} \Xi\left[W \gamma_{i} Z W^{\prime} \gamma_{j}^{\prime} Z^{\prime}\right]$

The functional $\Xi$ represents a multiple integration over $\xi\left(\phi-\phi^{\prime}, z, z^{\prime}\right) \mathrm{d}^{2} \phi \mathrm{d}^{2} \phi^{\prime} \mathrm{d} z \mathrm{~d} z^{\prime}$. In summary, the correlation for the shear can be written as

$$
\begin{aligned}
\operatorname{Cov}_{i j}\left(\hat{\gamma} ; \boldsymbol{\theta}, \boldsymbol{\theta}^{\prime}\right)=\frac{\sigma_{\epsilon}^{2} \delta_{i j}}{\langle Z\rangle^{2} \rho} \int_{\Omega} W(\boldsymbol{\theta}-\boldsymbol{\phi}) W\left(\boldsymbol{\theta}^{\prime}-\boldsymbol{\phi}\right) \mathrm{d}^{2} \phi \\
+\frac{\left\langle Z^{2}\right\rangle}{\langle Z\rangle^{2} \rho} \int_{\Omega} \gamma_{i}(\boldsymbol{\phi}) \gamma_{j}(\boldsymbol{\phi}) W(\boldsymbol{\theta}-\boldsymbol{\phi}) W\left(\boldsymbol{\theta}^{\prime}-\boldsymbol{\phi}\right) \mathrm{d}^{2} \phi \\
+\frac{1}{\langle Z\rangle^{2}} \Xi\left[W \gamma_{i} Z W^{\prime} \gamma_{j}^{\prime} Z^{\prime}\right]
\end{aligned}
$$

This expression, although rather complicated, already clearly shows the various contribution to the noise. The first term on the r.h.s., proportional to $\sigma_{\epsilon}^{2}$, is due to intrinsic scatter of source ellipticities. Lombardi \& Bertin (1998a) have already considered this term, and have also shown that because of its "diagonal" form (it is proportional to $\delta_{i j}$ and is a simple convolution of $W$ with itself) can be easily converted into a covariance for $\kappa$. Writing $\left\langle Z^{2}\right\rangle=\langle Z\rangle^{2}+\sigma_{Z}^{2}$, the second term on the r.h.s. can be thought as the sum of two terms. One term does not involve any redshift dependence and is a Poisson noise on the angular density of galaxies. The other term is proportional to $\sigma_{Z}^{2}$, the scatter of galaxies in redshift, and is a Poisson noise on the redshift distribution. These two terms can be better understood by two simple examples. Suppose that we observe some gravitational lens and that we make a shear map using the estimator of Eq. (4). Because of Poisson noise, in some region of the sky we could observe an overdensity of background galaxies, and thus obtain a larger than expected value for the shear in that region. On the other hand, in another region we could observe a "normal" density of galaxies, but their redshifts, because of Poisson noise, could be larger than expected. As a result, in this second region too we would over-estimate the shear. Situations such as the ones described contribute to the noise on $\hat{\gamma}$ by adding two sources of errors characterized by their dependence on $\gamma$ (in both cases considered above, in fact, no error is expected if $\gamma=0$ ). Finally, turning to the last term of Eq. (26), we note that it is clearly related to the two-point galaxy correlation function. Analogously to the second term of Eq. (26), this is actually composed of two contribution, one due to the angular correlation and one due to correlation in redshift (cf. Eqs. (23) and (24)).

From Eq. (26) we can also see that the first two noise terms are proportional to $1 / \rho$, while the last one is apparently independent of the density of background galaxies. This behavior is easily explained. Ellipticity and Poisson variances depends on single galaxies and thus increase linearly with $\rho$; hence, the factor $1 / \rho$ in Eq. (4) makes the final noise due to these sources proportional to $1 / \sqrt{\rho}$. On the other hand, the variance due to galaxy correlation is proportional to the number of pairs of galaxies and thus to $\rho^{2}$; the final correlation noise is then formally independent of $\rho$. We stress, however, that all noise terms depend on the galaxy redshift distribution. The density $\rho$ and the probability distribution $p_{z}(z)$ are intimately related. For example, deeper observations will increase $\rho$ but also the mean galaxy redshift. As a result, all noise terms do depend on the depth of the observation (see Sect. 7 for further comments on this point).

Looking again at Eq. (26), we can also obtain information about the typical correlation length for the shear, i.e. the largest angular separation between points that have correlated noise properties. The first term in this expression, in fact, suggests that the correlation length for $\hat{\gamma}$ is approximately twice the length scale of the weight function $W$; the same is true for the second term, while the third term has no "intrinsic" scale. This last point is basically due to the lack of a scale of the two-point correlation function as given by Eq. (11) (note that $d_{0}$ in this expression should be taken as a simple multiplicative coefficient rather than as a scale for $\xi$ ).

In principle, for a given lens, we could now evaluate the covariance of $\hat{\gamma}$ using the results obtained so far, and then convert it to the covariance of $\kappa$. Actually, in the limit of large fields $\Omega$, we can use the kernel (7) in Eq. (19), thus obtaining directly an expression for the covariance of $\kappa$ :

$$
\begin{aligned}
\operatorname{Cov}\left(\hat{\kappa} ; \boldsymbol{\theta}, \boldsymbol{\theta}^{\prime}\right)=\frac{\sigma_{\epsilon}^{2}}{\langle Z\rangle^{2} \rho} \int_{\Omega} \tilde{W}_{i}(\boldsymbol{\theta}-\boldsymbol{\phi}) \tilde{W}_{i}\left(\boldsymbol{\theta}^{\prime}-\boldsymbol{\phi}\right) \mathrm{d}^{2} \phi \\
+\frac{\left\langle Z^{2}\right\rangle}{\langle Z\rangle^{2} \rho} \int_{\Omega} \gamma_{i}(\boldsymbol{\phi}) \gamma_{j}(\boldsymbol{\phi}) \tilde{W}_{i}(\boldsymbol{\theta}-\boldsymbol{\phi}) \tilde{W}_{j}\left(\boldsymbol{\theta}^{\prime}-\boldsymbol{\phi}\right) \mathrm{d}^{2} \phi \\
+\frac{1}{\langle Z\rangle^{2}} \Xi\left[\tilde{W}_{i} \gamma_{i} Z \tilde{W}_{j}^{\prime} \gamma_{j}^{\prime} Z^{\prime}\right]
\end{aligned}
$$


Here, $\tilde{W}_{i}$ is a "modified weight", i.e. the convolution of $W$ with $\mathcal{D}_{i}^{\mathrm{KS}}$ :

$$
\begin{aligned}
\tilde{W}_{i}(\boldsymbol{\theta}) & =\int W(\boldsymbol{\theta}-\boldsymbol{\phi}) \mathcal{D}_{i}^{\mathrm{KS}}(\boldsymbol{\phi}) \mathrm{d}^{2} \phi \\
& =\mathcal{D}_{i}^{\mathrm{KS}}(\boldsymbol{\theta})\left[1-\left(1+\frac{|\boldsymbol{\theta}|^{2}}{2 \sigma_{W}^{2}}\right) \exp \left(-\frac{|\boldsymbol{\theta}|^{2}}{2 \sigma_{W}^{2}}\right)\right] .
\end{aligned}
$$

The last equality holds when a Gaussian weight function of the form (3) is used.

\section{Slowly varying shear}

The results obtained in the last section are exact but, unfortunately, difficult to interpret in general. In particular, it is not easy to estimate the order of magnitude of the expressions obtained, and it is also difficult to say which term makes the more important contribution in Eq. (26) (or, equivalently, Eq. (27)). In an important limit, however, the results obtained so far can be written in a much simpler and more useful form.

Suppose that the true shear field $\gamma(\boldsymbol{\theta})$ is slowly varying on the typical scale of the weight function $W$. In this limit, we can expand the shear to first-order and greatly simplify the integrals that involve $\gamma$ and $W$. The results obtained, though clearly not exact, represent an extremely useful starting point to evaluate the noise of the proposed shear estimates. Note that this technique can be successfully used to simplify the expressions of statistical estimates for the shear field, while is not directly applicable to expressions for the mass map because of the non-local dependence of $\kappa$ on $\gamma$ (or, equivalently, because of the infinite support of $\left.\mathcal{D}^{\mathrm{KS}}\right)$. Actually, we will still be able to obtain a finite form for the average value of $\hat{\kappa}(\boldsymbol{\theta})$ (see Sect. 3.1), but not for the covariance. However, if the noise properties of the shear are known, the covariance of $\hat{\kappa}$ can be evaluated using Eq. (19). Moreover, it is not unreasonable to assume that the covariance of $\hat{\kappa}$ is close to $\operatorname{Cov}(\hat{\gamma})$ [actually, if only the first term of Eq. (26) is taken into account, the two covariances are identical].

In the following we will always assume a symmetric weight function, i.e. such that $W\left(\boldsymbol{\theta}-\boldsymbol{\theta}^{\prime}\right)$ depends only on $\left|\boldsymbol{\theta}-\boldsymbol{\theta}^{\prime}\right|$. Moreover, the results obtained will also be specialized to the important case where $W$ is a Gaussian of the form (3). Finally, in order to numerically estimate the contribution of some terms to the noise, we will often refer to a "typical" model. The model is characterized by a lens at redshift $z_{\mathrm{d}}=0.3$ in an Einstein-de Sitter cosmological model. The normalized redshift probability distribution of source galaxies will be taken to be (see Brainerd et al. 1996)

$p_{z}(z)=\frac{\beta z^{2}}{\Gamma(3 / \beta) z_{0}^{3}} \exp \left[-\left(\frac{z}{z_{0}}\right)^{\beta}\right]$.

The constant $z_{0}$ determines the depth of the observations, while $\beta$ determines the steepness of the cutoff at high redshifts. The moments of this distribution are

$\left\langle z^{k}\right\rangle=z_{0}^{k} \frac{\Gamma((3+k) / \beta)}{\Gamma(3 / \beta)}$

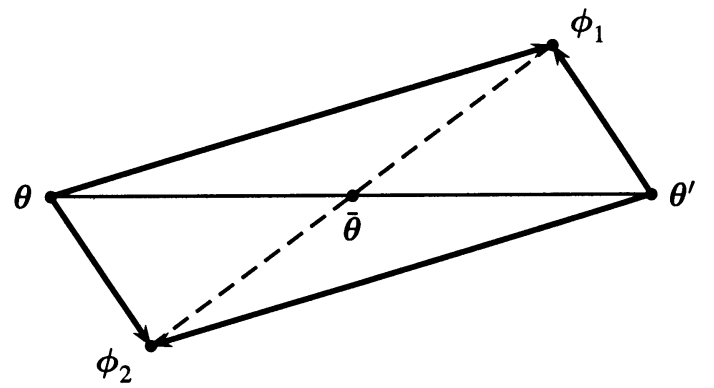

Fig. 1. Linear terms of the expansion of $\gamma$ around $\overline{\boldsymbol{\theta}}$ do not enter the expression for the covariance. In fact, the integrand of any linear term evaluated at the point $\phi_{1}$ is the opposite of the integrand evaluated at $\phi_{2}\left(\phi_{1}\right.$ and $\phi_{2}$ are such that $\left.\phi_{1}+\phi_{2}=2 \overline{\boldsymbol{\theta}}\right)$. In particular, the application of this symmetry to the liner expansion for the term $\gamma_{i}(\boldsymbol{\phi}) \gamma_{j}(\boldsymbol{\phi})$ leads to Eq. (34).

and the mode is

$z_{\text {mode }}=z_{0}\left(\frac{2}{\beta}\right)^{1 / \beta}$.

In the following, when not otherwise stated, we will use $\beta=1.5$ and $z_{0}=0.7$, so that $\langle z\rangle \simeq 1.05$ and $z_{\text {mode }} \simeq$ 0.848 .

\subsection{Average value}

If $\gamma\left(\boldsymbol{\theta}^{\prime}\right)$ does not change significantly when $\boldsymbol{\theta}^{\prime}$ is on the effective area of $W$, we can expand the shear around the point $\boldsymbol{\theta}$ :

$\gamma_{i}\left(\boldsymbol{\theta}^{\prime}\right) \simeq \gamma(\boldsymbol{\theta})+\left.\frac{\partial \gamma_{i}}{\partial \theta_{j}}\right|_{\boldsymbol{\theta}}\left(\theta_{j}^{\prime}-\theta_{j}\right)$

Inserting this expression into Eq. (15), and using the symmetry of $W$, we get

$\langle\hat{\gamma}(\boldsymbol{\theta})\rangle=\gamma(\boldsymbol{\theta}) \int_{\Omega} W\left(\boldsymbol{\theta}-\boldsymbol{\theta}^{\prime}\right) \mathrm{d}^{2} \theta^{\prime}=\gamma(\boldsymbol{\theta})$.

Equation (33) is clearly a rough approximation, since we already know that average value for the measured shear map is a smoothed version of the true shear. On the other hand, if the shear does not change significantly on the scale of $W$, we can safely take the result obtained; in more general cases it is, at least, an order-of-magnitude estimate.

\subsection{Covariance $(\xi=0)$}

We can in a similar way obtain an expression for the covariance of the shear using Eq. (26). The method used is similar to the one adopted above, with the only small difference that now the expansion for $\gamma$ will be performed around the mid-point $\overline{\boldsymbol{\theta}}=\left(\boldsymbol{\theta}+\boldsymbol{\theta}^{\prime}\right) / 2$ (see Fig. 1). We thus 
find

$$
\begin{aligned}
\operatorname{Cov}_{i j} & \left(\hat{\gamma} ; \boldsymbol{\theta}, \boldsymbol{\theta}^{\prime}\right)=\frac{1}{\langle Z\rangle^{2} \rho}\left[\sigma_{\epsilon}^{2} \delta_{i j}+\left\langle Z^{2}\right\rangle \gamma_{i}(\overline{\boldsymbol{\theta}}) \gamma_{j}(\overline{\boldsymbol{\theta}})\right] \\
\times & (W \star W)\left(\boldsymbol{\theta}-\boldsymbol{\theta}^{\prime}\right) \\
+ & \frac{\left\langle Z^{2}\right\rangle}{\langle Z\rangle^{2} \rho} \gamma_{i, i^{\prime}}(\overline{\boldsymbol{\theta}}) \gamma_{j, j^{\prime}}(\overline{\boldsymbol{\theta}}) \\
& \times \int_{\Omega} W(\boldsymbol{\theta}-\boldsymbol{\phi}) W\left(\boldsymbol{\theta}^{\prime}-\boldsymbol{\phi}\right)\left(\phi_{i^{\prime}}-\bar{\theta}_{i^{\prime}}\right)\left(\phi_{j^{\prime}}-\bar{\theta}_{j^{\prime}}\right) \mathrm{d}^{2} \phi
\end{aligned}
$$

where the $\operatorname{star}(\star)$ represents a convolution. In the case where $W$ is a Gaussian function we obtain in particular

$$
\begin{aligned}
\operatorname{Cov}_{i j}\left(\hat{\gamma} ; \boldsymbol{\theta}, \boldsymbol{\theta}^{\prime}\right)= & \frac{1}{4 \pi\langle Z\rangle^{2} \rho \sigma_{W}^{2}}\left[\sigma_{\epsilon}^{2} \delta_{i j}+\left\langle Z^{2}\right\rangle \gamma_{i}(\overline{\boldsymbol{\theta}}) \gamma_{j}(\overline{\boldsymbol{\theta}})\right. \\
& \left.+\frac{\left\langle Z^{2}\right\rangle \sigma_{W}^{2}}{2} \gamma_{i, k}(\overline{\boldsymbol{\theta}}) \gamma_{j, k}(\overline{\boldsymbol{\theta}})\right] \\
& \times \exp \left(-\frac{\left|\boldsymbol{\theta}-\boldsymbol{\theta}^{\prime}\right|^{2}}{4 \sigma_{W}^{2}}\right)
\end{aligned}
$$

This is the result for the covariance of $\hat{\gamma}$ in the slowly varying shear approximation. The fact that a finite expression has been obtained allows us to study the behavior of the various contributions to the noise. Let us discuss in detail the result obtained.

Equation (34) shows that the noise is proportional to a convolution of $W$ with itself. This suggests to define the effective area $A_{W}$ of the weight function, i.e. the area where $W(\phi)$ is significantly different from zero or, formally,

$A_{W}=\left[\int[W(\phi)]^{2} \mathrm{~d}^{2} \phi\right]^{-1}$.

For the Gaussian weight function we get $A_{W}=4 \pi \sigma_{W}^{2}$. We then note that the noise on $\hat{\gamma}$ given by Eq. (35) is proportional to $1 / N_{W}$, where $N_{W}=\rho A_{W}$ is the expected number of galaxies inside the effective area of $W$.

The ratio between the contributions to the noise arising from the shot-noise and the intrinsic scatter in ellipticities is of order $|\gamma(\boldsymbol{\theta})|^{2}\left\langle Z^{2}\right\rangle / \sigma_{\epsilon}^{2}$. Since $\sigma_{\epsilon} \simeq 0.25$, and $\left\langle Z^{2}\right\rangle \sim 1$, we see that the shot-noise is a relevant term unless the lens is very weak. More specifically, let us consider the error made on the component $i$ of the shear at $\boldsymbol{\theta}$. We have from Eq. (35) (no sum on $i$ )

$$
\begin{aligned}
\operatorname{Cov}_{i i}(\hat{\gamma} ; \boldsymbol{\theta}, \boldsymbol{\theta})= & \frac{1}{4 \pi\langle Z\rangle^{2} \rho \sigma_{W}^{2}}\left[\sigma_{\epsilon}^{2}+\left\langle Z^{2}\right\rangle\left[\gamma_{i}(\boldsymbol{\theta})\right]^{2}\right. \\
& \left.\quad+\frac{\left\langle Z^{2}\right\rangle \sigma_{W}^{2}}{2} \gamma_{i, k}(\boldsymbol{\theta}) \gamma_{i, k}(\boldsymbol{\theta})\right] \\
= & \Sigma_{\epsilon}^{2}+\Sigma_{\mathrm{P} 0}^{2}+\Sigma_{\mathrm{P} 1}^{2}
\end{aligned}
$$

where, for convenience, we have explicitely split the three contributions to the noise considered here. Using the lens model described above, we have $\langle Z\rangle \simeq 0.600810$ and $\sigma_{Z}^{2} \simeq$ 0.038347 . Hence, the expected error on $\hat{\gamma}$ due to the spread of source ellipticities is

$$
\begin{aligned}
\Sigma_{\epsilon}= & 0.016600\left(\frac{\sigma_{\epsilon}}{0.25}\right)\left(\frac{\sigma_{W}}{1 \operatorname{arcmin}}\right)^{-1} \\
& \times\left(\frac{\rho}{50{\text { gal } \operatorname{arcmin}^{-2}}^{-1 / 2}} .\right.
\end{aligned}
$$

The Poisson noise contributes with a term proportional to the shear,

$$
\begin{aligned}
\Sigma_{\mathrm{P} 0}= & 0.004196\left(\frac{\gamma}{0.1}\right)\left(\frac{\sigma_{W}}{1 \operatorname{arcmin}}\right)^{-1} \\
& \times\left(\frac{\rho}{50{\mathrm{gal} \operatorname{arcmin}^{-2}}^{-1 / 2}},\right.
\end{aligned}
$$

and with a term proportional to the derivatives of the shear,

$$
\Sigma_{\mathrm{P} 1}=0.002967\left(\frac{\gamma, j}{0.1 \operatorname{arcmin}^{-1}}\right)\left(\frac{\rho}{50 \mathrm{gal} \mathrm{arcmin}^{-2}}\right)^{-1 / 2} \text {. }
$$

Note that, surprisingly, this term does not depend on $\sigma_{W}$ (in this case, the angular scaling is given by the gradient of the shear). In conclusion, for a typical weak lens at redshift $z_{\mathrm{d}}=0.3$ we expect similar contributions from the intrinsic scatter of ellipticities and Poisson noise.

\subsection{Covariance $(\xi \neq 0)$}

Analytical calculations for the contributions to the covariance from the galaxy two-point correlation are definitely non-trivial. For this reason, we report here only the results of such calculations, and we refer to Appendix C for any details. Note that the calculations have been carried out only in the (important) case where $W$ is a Gaussian of the form (3). Moreover, sensible approximations have been used.

As discussed above (see Eqs. (11) and (12)), the twopoint correlation function is well approximated by a simple power-law in the physical separation of galaxies. Moreover, observations suggests that the exponent $\eta$ is very close to 2. This allows us to carry out calculations, obtaining (see Eq. (23))

$$
\begin{aligned}
C_{2}= & \frac{\pi^{3 / 2} d_{0}^{2}}{2\langle Z\rangle^{2} \sigma_{W}} \gamma_{i}(\overline{\boldsymbol{\theta}}) \gamma_{j}(\overline{\boldsymbol{\theta}}) I_{0}\left(\frac{|\boldsymbol{\varphi}|^{2}}{8 \sigma_{W}^{2}}\right) \exp \left(-\frac{|\boldsymbol{\varphi}|^{2}}{8 \sigma_{W}^{2}}\right) \\
& \times \int_{z_{d}}^{\infty} \frac{(1+z)^{\alpha}\left[p_{z}(z) Z(z)\right]^{2}}{D_{\mathrm{A}}(z) D_{\mathrm{P}}^{\prime}(z)} \mathrm{d} z,
\end{aligned}
$$

where $I_{0}$ is the modified Bessel function of first kind, $\boldsymbol{\varphi}=$ $\boldsymbol{\theta}^{\prime}-\boldsymbol{\theta}$, and $D_{\mathrm{A}}(z)$ and $D_{\mathrm{P}}(z)$ are, respectively, the angular diameter distance and the proper distance of an object at redshift $z$ [note that in Eq. (41) the derivative of $D_{\mathrm{P}}(z)$ is involved]. Note that, in Eq. (41), the smoothing length $\sigma_{W}$ has to be expressed in radians. Moreover, we have used as usual the notation $\overline{\boldsymbol{\theta}}$ for the mid-point between $\boldsymbol{\theta}$ and $\boldsymbol{\theta}^{\prime}$.

In the typical case discussed here of a lens at redshift $z_{\mathrm{d}}=0.3$ and using $d_{0}=5.4 h^{-1} \mathrm{Mpc}$ we have for the 
expected error due to the correlation of galaxies, $\Sigma_{2}=$ $\sqrt{C_{2}(\boldsymbol{\theta}, \boldsymbol{\theta})}$

$\Sigma_{2}=0.031173\left(\frac{\gamma}{0.1}\right)\left(\frac{\sigma_{W}}{1 \operatorname{arcmin}}\right)^{-1 / 2}$.

We must thus deduce that, at least for the case considered here, the galaxy two-point correlation function represents a major source of error in weak lensing estimates. A more detailed discussion on the relative contributions of the various sources of errors is delayed until Sect. 7 .

\section{The balanced shear estimator}

As already pointed out, the estimator (4) is very simple and traditionally has been the first one used (Kaiser \& Squires 1993). On the other hand, this estimator has a rather large noise and in particular suffers from Poisson noise, due to the fact that the normalization factor is constant both on the density of galaxies $(1 / \rho)$ and on the redshift distribution of sources $(1 /\langle Z\rangle)$.

In this section we will study in detail the estimator (5) similarly to what we have already done in Sects. 3 and 4 for the estimator (4). However, since now the estimator is non-linear on the observed positions of galaxies, an analytical evaluation of the average values and errors for this estimator would be hopeless without any approximations. For this reason we will linearize Eq. (5) around the average values of the random variables involved. This technique, often adopted in statistics, is briefly described in Appendix A. Moreover, to simplify the discussion, we will mostly report here only results, referring to Appendices B and $\mathrm{C}$ for the derivations.

\subsection{Linear approximation}

Let us call $N$ and $D$ the numerator and denominator of Eq. (5), so that $\hat{\gamma}_{i}=N_{i} / D$. Using the linear approximation (see Appendix A), we can write the expectation value of $\hat{\gamma}_{i}$ as

$\left\langle\hat{\gamma}_{i}\right\rangle \simeq \frac{\left\langle N_{i}\right\rangle}{\langle D\rangle}-\frac{1}{\langle D\rangle^{2}} \operatorname{Cov}\left(N_{i} D\right)+\frac{\left\langle N_{i}\right\rangle}{\langle D\rangle^{3}} \operatorname{Cov}(D D)$

The last two terms on the r.h.s. represent second-order corrections and will often be ignored. The covariance of $\hat{\gamma}$ can be written as $\operatorname{Cov}_{i j}\left(\hat{\gamma} ; \boldsymbol{\theta}, \boldsymbol{\theta}^{\prime}\right)$, i.e. as a $2 \times 2$ matrix which depends on two spatial coordinates $\boldsymbol{\theta}$ and $\boldsymbol{\theta}^{\prime}$ (see Lombardi \& Bertin 1998a). Omitting the two arguments $\boldsymbol{\theta}$ and $\boldsymbol{\theta}^{\prime}$, we can write

$$
\begin{gathered}
\operatorname{Cov}_{i j}(\hat{\gamma})=\frac{1}{\langle D\rangle\left\langle D^{\prime}\right\rangle} \operatorname{Cov}\left(N_{i} N_{j}^{\prime}\right)-\frac{\left\langle N_{i}\right\rangle}{\langle D\rangle^{2}\left\langle D^{\prime}\right\rangle} \operatorname{Cov}\left(D N_{j}^{\prime}\right) \\
-\frac{\left\langle N_{j}^{\prime}\right\rangle}{\langle D\rangle\left\langle D^{\prime}\right\rangle^{2}} \operatorname{Cov}\left(N_{i} D^{\prime}\right)+\frac{\left\langle N_{i}\right\rangle\left\langle N_{j}^{\prime}\right\rangle}{\langle D\rangle^{2}\left\langle D^{\prime}\right\rangle^{2}} \operatorname{Cov}\left(D D^{\prime}\right)
\end{gathered}
$$

Here we have used the prime to denote quantities which must be evaluated at $\boldsymbol{\theta}^{\prime}$ rather then at $\boldsymbol{\theta}$.

As a general rule, the linear approximation can be used when the denominator $D$ is expected to have a small (relative) variance. This is true if the expected number of galaxies inside the effective area of the smoothing function $W$ is much larger than 1.

We also note that in the linear approximation, the expression for the covariance for $\gamma$ depends on the two-point galaxy correlation function only, and not on higher-order correlation functions. This would not be true without the linear approximation.

\subsection{Slowly varying shear}

Similarly to what we have seen above for the unbalanced estimator (4), the statistical properties of the balanced estimator (5) are better understood in the limit where the shear is slowly changing on the effective area of the weight function. In this case, as shown in detail in Appendix C, we can explicitely write the expectation value and the covariance of $\hat{\gamma}$.

Regarding the expectation value of $\hat{\gamma}$, we obtain a result similar to Eq. (33), i.e. $\langle\hat{\gamma}(\boldsymbol{\theta})\rangle=\gamma(\boldsymbol{\theta})$. In other words, in the slowly varying shear approximation, the estimator (5) is unbiased. Note that in more general cases we still recover Eq. (15), i.e. the average value for the estimator $\hat{\gamma}$ is the true shear $\gamma$ convolved with the weight function ${ }^{1}$. Finally, since Eq. (15) holds, we can immediately deduce that Eq. (16) holds as well.

Turning to covariances, we can finally see the advantages of using a balanced estimator for the shear. In fact, in the slowly varying shear approximation and neglecting the two-point correlation function, we obtain for the covariance of $\hat{\gamma}$, in the case where $W$ is a Gaussian function,

$$
\begin{aligned}
& \operatorname{Cov}_{i j}\left(\hat{\gamma} ; \boldsymbol{\theta}, \boldsymbol{\theta}^{\prime}\right)=\frac{1}{4 \pi\langle Z\rangle^{2} \rho \sigma_{W}^{2}}\left[\sigma_{\epsilon}^{2} \delta_{i j}+\sigma_{Z}^{2} \gamma_{i}(\overline{\boldsymbol{\theta}}) \gamma_{j}(\overline{\boldsymbol{\theta}})\right. \\
& \left.\quad+\left(\frac{\left\langle Z^{2}\right\rangle \sigma_{W}^{2}}{2} \delta_{i^{\prime} j^{\prime}}-\frac{\langle Z\rangle^{2}}{4} \varphi_{i^{\prime}} \varphi_{j^{\prime}}\right) \gamma_{i, i^{\prime}}(\overline{\boldsymbol{\theta}}) \gamma_{j, j^{\prime}}(\overline{\boldsymbol{\theta}})\right] \\
& \quad \times \exp \left(-\frac{|\phi|^{2}}{4 \sigma_{W}^{2}}\right)
\end{aligned}
$$

with $\sigma_{Z}^{2}=\left\langle Z^{2}\right\rangle-\langle Z\rangle^{2}$. This expression should be compared with the analogous equation obtained for the estimator (4), namely Eq. (35). The basic difference is given by the factor $\left\langle Z^{2}\right\rangle$ which replaces $\sigma_{Z}^{2}$ of Eq. (45) or, in other words, by the presence of an extra term $\langle Z\rangle^{2}$ in Eq. (35). This extra term is precisely due to Poisson noise on the angular coordinate $\boldsymbol{\theta}$; analogously, the term $\sigma_{Z}^{2}$ left can be interpreted as Poisson noise in redshift. In order to better appreciate this point, it is interesting to evaluate the expected error of the balanced estimator in the lens model described above. In this case we have $\langle Z\rangle \simeq 0.600810$ and $\sigma_{Z}^{2} \simeq 0.038347$. Hence, the use of a balanced estimator reduces the "constant" component of

\footnotetext{
1 Actually, a rigorous calculation shows that the average value for $\hat{\gamma}$ is the true shear $\gamma$ convolved with a kernel slightly different from $W$. The kernel quickly converges to the weight function when the number of effective galaxies is large (see Lombardi \& Schneider 2001).
} 
Poisson noise by about a factor 4 (i.e. a factor 16 in variance). More explicitely, the contribution of the redshift Poisson noise is given by

$$
\begin{aligned}
\Sigma_{\mathrm{P} 0}= & 0.001300\left(\frac{\gamma}{0.1}\right)\left(\frac{\sigma_{W}}{1 \operatorname{arcmin}}\right)^{-1} \\
& \times\left(\frac{\rho}{50{\text { gal } \operatorname{arcmin}^{-2}}^{-1 / 2} .}\right.
\end{aligned}
$$

This expression replaces (39). Note that, in contrast, the Poisson noise due to shear variations [represented by the second line in Eq. (45)], is only partially reduced by the balanced estimator. In particular, if $\boldsymbol{\varphi}=\mathbf{0}$, no improvement is obtained and Eq. (40) can still be used to have an estimate of this noise source.

If the two-point galaxy correlation is taken into account using the model (12), we have a supplementary term in the covariance. For the lens considered here, this term is of the order (see Appendix C)

$\Sigma_{2}=0.007152\left(\frac{\gamma}{0.1}\right)\left(\frac{\sigma_{W}}{1 \operatorname{arcmin}}\right)^{-1 / 2}$.

Comparing this result with Eq. (42), we see that the use of the balanced estimator has significantly reduced the noise due to the clustering of galaxies.

\section{Numerical estimates}

In the previous sections, we have obtained estimates for the various contributions to the noise of the shear using several approximations, namely the weak lensing limit, the slowly varying shear (Sect. 4), and the linear expansion of the estimators (Sect. 5.1). Among these approximations, probably the most critical one is that of the slowly varying shear. Hence, in order to test the reliability of the results obtained so far, we have numerically evaluated the noise on the shear estimator (4) in a specific case. Note that, instead, it is extremely difficult to test directly the results for the balanced estimator without using the linear approximation described in Sect. 5.1. In this case, in fact, we would need to average over the whole galaxy positions $\left\{\boldsymbol{\theta}^{(n)}\right\}$, using also a probability distribution $p\left(\boldsymbol{\theta}^{(1)}, \boldsymbol{\theta}^{(2)}, \ldots, \boldsymbol{\theta}^{(N)}\right)$ which includes effects of $n$-point correlation (actually, even $N$, the total number of galaxies, should be taken as a random variable with Poisson distribution). Clearly, such an analysis would be prohibitive.

We have considered a lens at redshift $z_{\mathrm{d}}=0.3$ with an axisymmetric profile (see Fig. 2):

$\kappa(\boldsymbol{\theta})=\frac{\kappa_{0} \theta_{\mathrm{t}}}{\theta_{\mathrm{t}}-\theta_{\mathrm{c}}} f\left(|\boldsymbol{\theta}| / \theta_{\mathrm{c}}\right)-\frac{\kappa_{0} \theta_{\mathrm{c}}}{\theta_{\mathrm{t}}-\theta_{\mathrm{c}}} f\left(|\boldsymbol{\theta}| / \theta_{\mathrm{t}}\right)$,

where $f(x)$ is the dimensionless function

$f(x)=\frac{1}{\sqrt{1+x^{2}}}$.

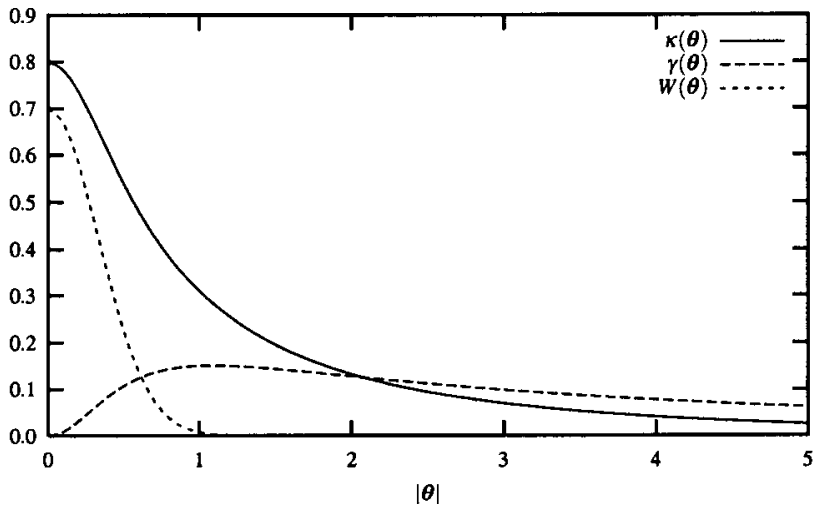

Fig. 2. The lens mass profile $\kappa(\boldsymbol{\theta})$ and the lens shear $\gamma(\boldsymbol{\theta})$ at different radii. The plot also shows the Gaussian weight function $W(\boldsymbol{\theta})$ used. Notice that $w(\boldsymbol{\theta})$ in this plot is not normalized.

Note that $f(x) \propto 1 / x$ for large $x, \kappa(\boldsymbol{\theta}) \propto 1 /|\boldsymbol{\theta}|$ for $\theta_{\mathrm{c}} \ll$ $|\theta| \ll \theta_{\mathrm{t}}$, while $\kappa(\boldsymbol{\theta}) \propto 1 /|\boldsymbol{\theta}|^{3}$ for $|\boldsymbol{\theta}| \gg \theta_{\mathrm{t}}$. For this lens, the shear is easily obtained: its modulus is

$\gamma(\boldsymbol{\theta})=\frac{\kappa_{0} \theta_{\mathrm{t}}}{\theta_{\mathrm{t}}-\theta_{\mathrm{c}}} g\left(|\boldsymbol{\theta}| / \theta_{\mathrm{c}}\right)-\frac{\kappa_{0} \theta_{\mathrm{c}}}{\theta_{\mathrm{t}}-\theta_{\mathrm{c}}} g\left(|\boldsymbol{\theta}| / \theta_{\mathrm{t}}\right)$,

with

$g(x)=\frac{\left[\sqrt{1+x^{2}}-1\right]^{2}}{x^{2} \sqrt{1+x^{2}}}$.

The shear decreases as $\gamma(\boldsymbol{\theta}) \propto 1 /|\boldsymbol{\theta}|^{2}$ for large $|\boldsymbol{\theta}|$ (see Fig. 2).

Using this lens model, we have carried out the integrations of the various terms of Eq. (26) numerically, and compared the results obtained with the analytical predictions of Sect. 4. Table 1 shows the relevant parameters used in the calculations. The results obtained are summarized in Figs. 3 and 4.

Figure 2 shows the lens profile together with the tangential shear. In this figure we have also plotted the weight function $W(\boldsymbol{\theta})$ used in the calculations. As shown by Fig. 2, the slowly varying shear approximation is not really justified in this case, since the lens shear shows significant

Table 1. Relevant parameters used for the numerical integration.

\begin{tabular}{ccl}
\hline Variable & Value & Comment \\
\hline$z_{\mathrm{d}}$ & 0.3 & Lens redshift \\
$\theta_{\mathrm{c}}$ & $30^{\prime \prime}$ & Lens core radius (Eq. (48)) \\
$\theta_{\mathrm{t}}$ & $5^{\prime}$ & Lens truncation radius (Eq. (48)) \\
$\kappa_{0}$ & 0.8 & Lens central density (Eq. (48)) \\
$\rho$ & $50 \operatorname{arcmin}^{-2}$ & Density of galaxies \\
$\sigma_{\epsilon}$ & 0.25 & Intrinsic ellipticity scatter \\
$z_{0}$ & 0.7 & Redshift distr. parameter (Eq. (29)) \\
$\beta$ & 1.5 & Redshift distr. slope (Eq. (29)) \\
$d_{0}$ & $5.4 h \mathrm{Mpc}$ & Galaxy correlation length (Eq. (12)) \\
$\eta$ & 1.77 & Galaxy correlation slope (Eq. (12)) \\
$\alpha$ & 2 & Galaxy correlation redshift \\
$\sigma_{W}$ & $20^{\prime \prime}$ & dependence (Eq. (12)) \\
\hline
\end{tabular}




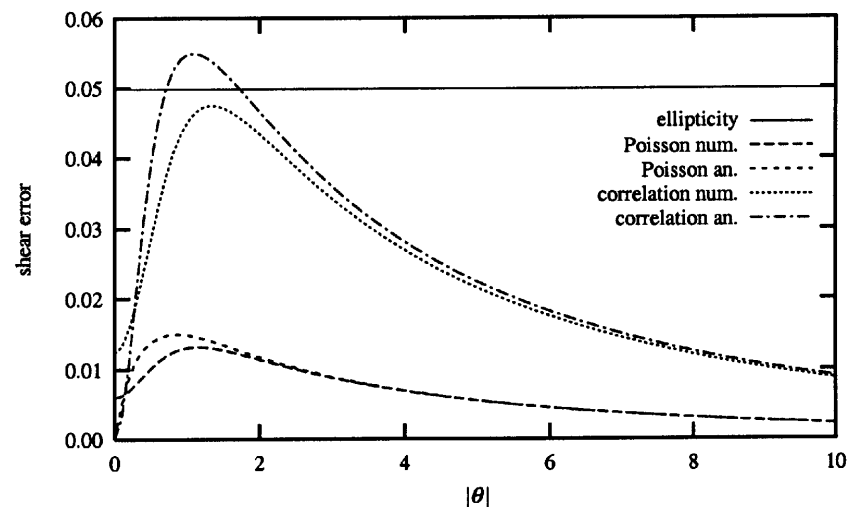

Fig. 3. The different contributions to the shear error (i.e., square root of variance) at different radii. Note that the analytical estimates, obtained from the expressions of Sect. 4, are in very good agreement with the numerical results.

deviations from linearity on the scale of the weight function. However, we find interesting to consider this situation in order to better test our analytical approximations.

Figure 3 shows, for different radii, the expected errors on the shear coming from different sources of noise. The horizontal line is the intrinsic ellipticity noise, which clearly is constant on the whole field. Note that the analytical estimate for this noise source is exact. As shown by Fig. 3, correlation of galaxies is the main source of noise at $|\boldsymbol{\theta}| \simeq 1^{\prime}$, while Poisson noise is relatively unimportant (see Sect. 7 below for further comments on this point). The analytical approximations given in Sect. 4 perform surprisingly well, with deviations below $10 \%$ for most radii. The larger deviations observed around $|\boldsymbol{\theta}|=1^{\prime}$ are expected, since at this radius the shear deviates significantly from the linear approximation (the same is true around the origin, where the shear vanishes). We also point out that the analytical estimate for the correlation noise is slightly less accurate than the one for Poisson noise mainly because Eq. (41) is a zero-order estimate (in the sense that terms containing shear derivatives have not been considered in this equation).

Figure 4 shows similar results for the noise on the mass map $\kappa(\boldsymbol{\theta})$. The numerical integrations have been performed using Eq. (27), while the analytical estimates are the same as for the shear (i.e., we have assumed that the noise on the mass map is the same as the noise on the shear). Note that, except at small radii, the analytical estimates for the shear provide an accurate measure of the noise on the mass map. In reality, for more complicated lenses this property is not verified at the degree of precision of Fig. 4; nevertheless, the analytical expressions for the shear noise are always a good order-of-magnitude estimate for the noise in the mass map.

\section{Impact on observations}

So far we have studied the statistical properties of two shear estimators from a purely analytical point of view. Clearly, the interest of our results lies on the consequences

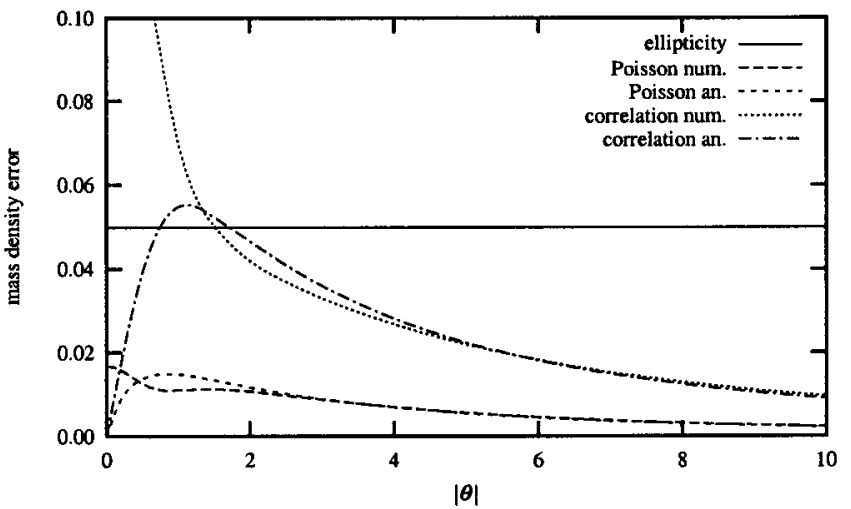

Fig. 4. The different contributions to the mass noise at different radii. Although the analytical curves refer to the shear noise, they provide a very good estimate for all radii larger than $1^{\prime}$. The scale of this figure is the same as Fig. 3; the curve for the numerical evaluation of the correlation noise reaches about 0.67 at the origin.

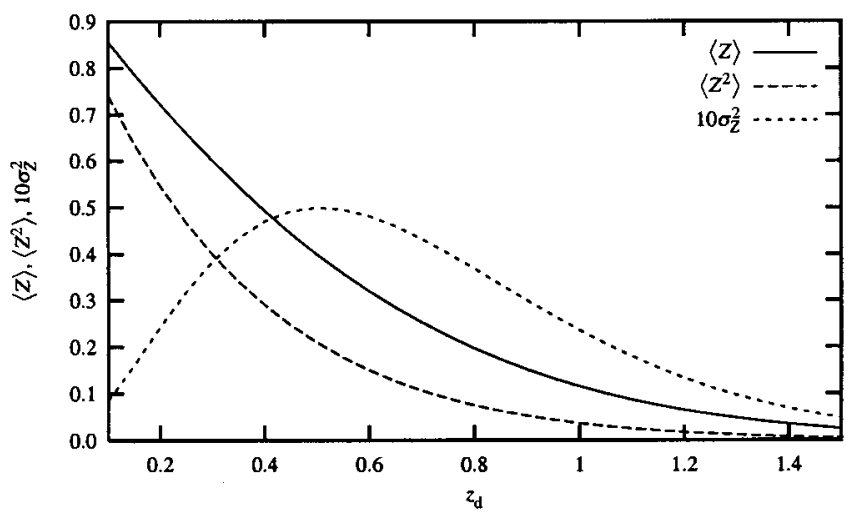

Fig. 5. The mean cosmological weight and its moments as a function of the lens redshift $z_{\mathrm{d}}$. Note that the quantity $\sigma_{Z}$ has a peak around $z_{\mathrm{d}}=0.5$.

of the various noise sources on weak lensing studies. In this section we consider the expressions obtained in Sects. 4 and 5 from a more practical point of view, with particular attention to their dependence on critical observational parameters (such as the redshift and the strength of the lens, the density of galaxies, and their mean redshift).

As already stressed, various sources of noise contribute to the expected error of both estimators. In particular, we have found three main contributions:

1. the intrinsic scatter of source ellipticities;

2. the Poisson noise, both on the positions of galaxies and on their redshift;

3. the clustering of galaxies, characterized by the twopoint correlation function $\xi$.

Depending on some key parameters, these sources of noise can have different relative importance for the total error of the measured shear, and thus of the reconstructed lens mass map.

For weak lenses the leading term is the intrinsic scatter of ellipticities. In fact, the Poisson noise and the noise due to the clustering of galaxies are proportional to the shear, 


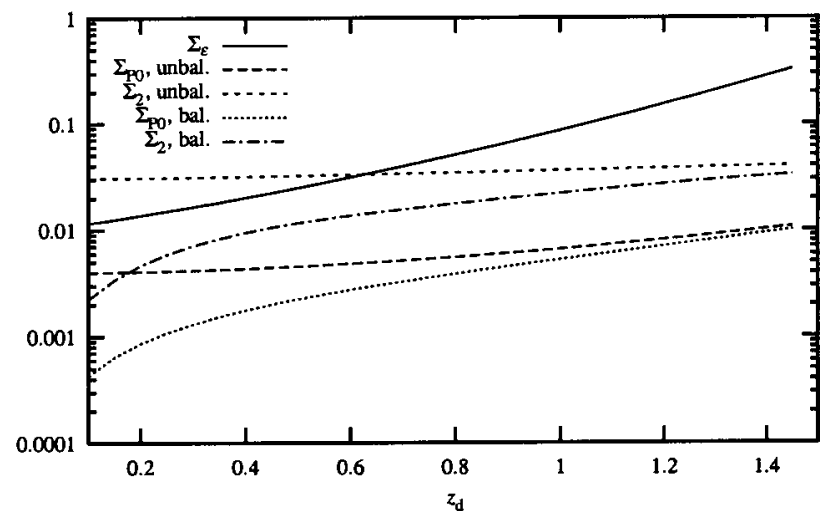

Fig. 6. Typical errors on the shear due to various sources of noise. The plot shows the numerical factors in front of some equations describing errors on $\hat{\gamma}$. For all graphs we assumed $\rho=50$ gal $\operatorname{arcmin}^{-2}, \sigma_{W}=1$ arcmin, and $\gamma=0.1$. For the estimator (4), Eq. (38) gives the error due to the intrinsic scatter of source ellipticities $\Sigma_{\epsilon}$ (assuming $\sigma_{\epsilon}=0.25$ ), Eq. (39) the Poisson noise $\Sigma_{\mathrm{P} 0}$ (both in redshift and in position), and Eq. (42) the noise due to clustering of galaxies $\Sigma_{2}$; for the estimator (5), the noise due to source ellipticities is still given by Eq. (38), the Poisson noise (now only on redshift) is given by Eq. (46), and the clustering noise by Eq. (47). Note that the relative importance of error source depends critically on the shear $\gamma$. For example, for a lens with $\gamma=0.3$ the clustering noise for the balanced estimator would be larger than the intrinsic ellipticity noise in the redshift range $0.2<z_{\mathrm{d}}<0.9$.

and thus are expected to have a negligible impact on very weak lenses. We note that this point is very important for cosmic shear studies.

The noise due to galaxy clustering shows rather surprising properties if compared to other sources of errors. As already pointed out in Sect. 3.2, ellipticity and Poisson noise decrease with the density of background sources as $1 / \sqrt{\rho}$; in contrast, the noise due to clustering is formally independent of the density of galaxies. Hence, this source of error is expected to be particularly important in deep observations and can represent a major limitation for weak lensing studies in the near future. In reality, deeper observations also imply a change of the redshift distribution $p_{z}$ and, in particular, an increase of $\langle z\rangle$. As a result, the correlation error decreases for deep observations, but not as fast as other sources of noise.

Table 2 reports typical figures for the various noise terms as a function of the density of background galaxies. Calculations for that table have been carried out using $\sigma_{\epsilon}=0.25, \sigma_{W}=1$ arcmin, and a lens shear $\gamma=0.1$. In order to simulate the increase of the mean redshift for deep observations, we have used a galaxy redshift probability distribution $p_{z}(z)$ of the form of Eq. (29), with $\beta=1.5$ and $z_{0}$ dependent on the density $\rho$. In particular, we have used $z_{0}=0.6$ for $\rho=25 \operatorname{arcmin}^{-2}, z_{0}=0.7$ for $\rho=50 \operatorname{arcmin}^{-2}$, and $z_{0}=0.8$ for $\rho=100 \operatorname{arcmin}^{-2}$. Looking at Table 2 , we find surprisingly that the ellipticity noise decreases as $\rho^{-0.60}$ instead of as $\rho^{-1 / 2}$, as suggested by Eq. (38). The 0.1 increase in the logarithmic slope of $\Sigma_{\epsilon}$ is due to the change in the galaxy redshift distribution
Table 2. Noise terms as a function of the observation depth. The table reports the expected noise contributions (square root of variance contributions) from various sources of noise. The last column gives the logarithmic slope of the respective noise term in terms of $\rho$, i.e. the constant $k$ in the relation $\Sigma_{X} \propto \rho^{k}$.

\begin{tabular}{|c|c|c|c|c|}
\hline \multirow[t]{2}{*}{ Noise term } & \multicolumn{3}{|c|}{ Density $\rho\left(\operatorname{arcmin}^{-2}\right)$} & \multirow[t]{2}{*}{$k$} \\
\hline & 25 & 50 & 100 & \\
\hline $\begin{array}{r}\text { Ellipticity noise } \Sigma_{\epsilon} \\
\text { Un }\end{array}$ & $\begin{array}{l}0.025689 \\
\text { balanced e }\end{array}$ & $\begin{array}{l}0.016600 \\
\text { estimator }\end{array}$ & 0.010996 & -0.60 \\
\hline Poisson noise $\Sigma_{\mathrm{P} 0}$ & 0.006039 & 0.004196 & 0.002933 & -0.52 \\
\hline Correlation noise $\Sigma_{2}$ & $\begin{array}{l}0.033567 \\
\text { alanced es }\end{array}$ & $\begin{array}{l}0.031173 \\
\text { timator }\end{array}$ & 0.029393 & -0.10 \\
\hline Poisson noise $\Sigma_{\mathrm{P} 0}$ & 0.002155 & 0.001300 & 0.000803 & -0.72 \\
\hline Correlation noise $\Sigma_{2}$ & 0.009067 & 0.007152 & 0.005812 & -0.33 \\
\hline
\end{tabular}

considered here, and in particular is related to the coefficient $1 /\langle Z\rangle^{2}$ in Eq. (37). In contrast, the Poisson noise $\Sigma_{P 0}$ for the unbalanced estimator almost exactly keeps the $\rho^{-1 / 2}$ dependence. This is due to a cancellation effect in Eq. (37), between $\langle Z\rangle^{2}$ in the denominator and $\left\langle Z^{2}\right\rangle$ in the numerator. The correlation noise $\Sigma_{2}$ for the unbalanced estimator is found to be basically constant with $\rho$; again, the 0.1 difference between the expected logarithmic slope $\left(\Sigma_{2} \propto \rho^{0}\right)$ and the observed one $\left(\Sigma_{2} \propto \rho^{-0.1}\right)$ can be attribute to a $1 /\langle Z\rangle^{2}$ factor (see Eq. (41)).

Turning to the balanced estimator, we immediately note a more pronounced dependence of the noise terms on $\rho$. The Poisson noise decreases as $\rho^{-0.72}$, i.e. with a logarithmic slope 0.22 larger than suggested by Eq. (46). We can certainly attribute 0.10 increase to the factor $1 /\langle Z\rangle^{2}$ in Eq. (45); the remaining 0.12 increase is due to $\sigma_{Z}^{2}$, which decreases as the bulk of sources is shifted towards high redshift. The correlation noise is found to have a density dependence $\rho^{-0.33}$. It is interesting to note that for both Poisson noise and correlation noise we gain an exponent -0.2 with respect to the unbalanced estimator.

For both estimators, the correlation noise is found to be significant (we recall that calculations for Table 2 have been carried out assuming a shear of only 0.1 ). Fortunately, as we will see in the next section, this source of noise disappears if we have information on the redshifts of galaxies (e.g., though photometric redshifts) and if we make use of this data. A second important point is the dependence of the noise terms on the lens redshift. Figure 6 gives a numerical estimate for the various sources of error as a function of $z_{\mathrm{d}}$. All errors increase with redshift, and in particular the error due to source ellipticities is quickly increasing. In fact, the lensing signal decreases as $1 /\langle Z\rangle$, thus is strongly dependent on the lens redshift (we recall that $Z(z)=0$ for $\left.z \leq z_{\mathrm{d}}\right)$. Other sources of noise are less dependent on the redshift of the lens. It is also interesting to observe that the largest contribution to Poisson and clustering noise comes from the angular dependence [compare the curve $\Sigma_{\mathrm{P} 0}$ for the unbalanced estimator, which is relative to Poisson noise in angular positions and redshifts of galaxies, with the curve $\Sigma_{\mathrm{P} 0}$ for the balanced estimator, 
which includes only redshift Poisson noise; similarly for the curves $\Sigma_{2}$. This last point is basically due to the "compression" of distances in the redshift space. Assuming that the redshift is only cosmological, two galaxies at slightly different redshifts (say $\Delta z=0.01$ ) can actually be several Mpc away, so that only weak correlations are expected for such galaxies. On the other hand, their cosmological weight is basically the same, and thus no extra noise on the shear is expected. We also stress here the different angular dependence of the clustering noise, which decreases just as $1 /\left|\boldsymbol{\theta}-\boldsymbol{\theta}^{\prime}\right|$ at large radii (see Sect. C.4), i.e. it introduces a long-range correlation on the map (in contrast, ellipticity and Poisson noise correlation decrease very rapidly with $\left|\boldsymbol{\theta}-\boldsymbol{\theta}^{\prime}\right|$, as a Gaussian).

Finally, it is worth to mention a general technique often used to estimate errors of weak lensing studies, namely bootstrapping. This method is based on the generation of many datasets from the observed data to be used for the determination of errors. Suppose that we observe $N$ galaxies with ellipticities $\left\{\epsilon^{(n)}\right\}$ and positions $\left\{\boldsymbol{\theta}^{(n)}\right\}$. We can then construct a new galaxy catalog by randomly drawing $N$ galaxies from the original catalog, with replacement. Because of the replacements, we are allowed to use a galaxy, which is represented here by $(\boldsymbol{\theta}, \epsilon)$, more that once. We can then use the new catalog to perform a weak lensing mass reconstruction. By repeating the whole process several times, we eventually obtain the noise properties of the mass map. Bootstrapping is a very simple and reasonably robust method to obtain error estimates, but unfortunately relies on the assumption of independent data. In our specific case, because of the spatial correlation of galaxies, we are not really dealing with independent data, and thus, at least in principle, the use bootstrapping is not fully justified. Moreover, the use of analytical expressions provided here for the noise estimate has the advantage of showing clearly the different noise contributions and of allowing us to predict the expected errors without using any specific dataset.

\section{Sources with known redshifts}

When some kind of knowledge about the redshift of each galaxy is available, for example through photometric redshifts, we can take advantage of the extra information to reduce the error on the shear (and thus on the mass map) by adopting some improved estimator.

Suppose that measured redshifts $\left\{\hat{z}^{(n)}\right\}$ for source galaxies are available, together with their expected errors. Then, assuming a particular cosmological model, we can convert these redshifts into the corresponding cosmological weights $\left\{\hat{Z}^{(n)}\right\}$. If the estimates of the redshifts are unbiased and their expected errors small, we can take the cosmological weights unbiased, i.e. with some "symmetric" scatter around the true weights.

In order to take into account errors on measured redshifts, we introduce $p_{\hat{z}}(\hat{z} \mid z)$, the Baysian probability distribution for $\hat{z}$. This function gives the probability of measuring $\hat{z}$ when the real redshift is $z$. Note that we assume that the probability distribution for $\hat{z}$ depends only on $z$, the true redshift. Referring to photometric redshifts, we observe that, on one hand, this approximation neglects some key points, such as the dependence of the error on the photometry (and thus luminosity) of the galaxy; on the other hand, since, except for the redshift $z$, we are dealing with quantities which can be taken to be uncorrelated with the measured redshift $\hat{z}$ of a galaxy $\left(\boldsymbol{\theta}\right.$ and $\epsilon^{\mathrm{s}}$ have clearly nothing to do with $\hat{z}$ ), we can safely simplify the discussion using $p_{\hat{z}}(\hat{z} \mid z)$. When performing averages, we need to include the new random variable $\hat{z}$. As a result, we have to modify Eqs. (8) and (9) with the replacement

$p_{z}(z) \mapsto \int_{0}^{\infty} p_{\hat{z}}(\hat{z} \mid z) \mathrm{d} \hat{z}$.

Note that in terms of the probability distribution $p_{\hat{z}}(\hat{z} \mid z)$, the condition of unbiased cosmological weights estimates can be written as

$\langle\hat{Z}\rangle \equiv \int p_{\hat{z}}(\hat{z} \mid z) Z(\hat{z}) \mathrm{d} \hat{z}=Z(z)$.

In order simplify the discussion, in the following we will assume that the expected error on the cosmological weight is a simple function of the redshift of the galaxy, which we write $\sigma_{\hat{Z}}(z)$ :

$\sigma_{\hat{Z}}^{2}(z)=\int_{0}^{\infty} p_{\hat{z}}(\hat{z} \mid z)[Z(\hat{z})-Z(z)]^{2} \mathrm{~d} \hat{z}$.

We will often refer to the variance of measured redshifts. This quantity is defined as

$\sigma_{\hat{Z}}^{2} \equiv \int_{0}^{\infty} p_{z}(z) \sigma_{\hat{Z}}^{2}(z) \mathrm{d} z$.

We stress that this variance depends on both the errors in redshifts and on the source redshift probability distribution. To give an example of the precision that can be reached by photometric redshifts when accurate photometry is available, we note that for the Hubble Deep Field North a scatter of the order of $0.07(1+z)$, without outliers and bias, has been reached (Benitez 2000).

We will now investigate two possible uses of the quantities $\left\{\hat{Z}^{(n)}\right\}$ for shear estimators. Our starting model will be the estimator of Eq. (5), since the estimator (4) has already been proven to have a larger variance.

\subsection{Unweighted estimator}

In Sect. 5 we have seen that the use of a balanced estimator on the source positions completely eliminates Poisson noise due to the galaxy positions and significantly reduces the correlation noise. Since estimates for the cosmological weights are now available, we can now think of replacing the average $\langle Z\rangle$ in the denominator of Eq. (5) with the measured values for the cosmological weight. This leads to the new shear estimator

$\hat{\gamma}(\boldsymbol{\theta})=\frac{\sum_{n=1}^{N} \epsilon^{(n)} W\left(\boldsymbol{\theta}-\boldsymbol{\theta}^{(n)}\right)}{\sum_{n=1}^{N} \hat{Z}^{(n)} W\left(\boldsymbol{\theta}-\boldsymbol{\theta}^{(n)}\right)}$. 
This estimator is called "unweighed" since we are not taking advantage of the redshift information to weight galaxies (a weighted estimator will be discussed below in Sect. 8.2). Using a technique very similar to the one discussed above, we can study the statistical properties of this estimator, and in particular its mean and variance.

Regarding the mean, in the linear approximation this estimator behaves exactly as the estimators considered so far, i.e. Eq. (15) holds. In the slowly varying shear approximation, moreover, we have $\langle\hat{\gamma}(\boldsymbol{\theta})\rangle=\gamma(\boldsymbol{\theta})$. We stress that a key point in deriving these results is Eq. (53), i.e. the condition of unbiased cosmological weights.

Using the slowly varying shear approximations and in case of a vanishing two-point galaxy correlation function, the covariance of $\hat{\gamma}$ is

$$
\begin{aligned}
& \operatorname{Cov}_{i j}\left(\hat{\gamma} ; \boldsymbol{\theta}, \boldsymbol{\theta}^{\prime}\right)=\frac{1}{4 \pi\langle Z\rangle^{2} \rho \sigma_{W}^{2}}\left[\sigma_{\epsilon}^{2} \delta_{i j}+\sigma_{\hat{Z}}^{2} \gamma_{i}(\boldsymbol{\theta}) \gamma_{j}\left(\boldsymbol{\theta}^{\prime}\right)\right. \\
& \left.+\left(\frac{\left\langle Z^{2}\right\rangle \sigma_{W}^{2}}{2} \delta_{i^{\prime} j^{\prime}}-\frac{\langle Z\rangle^{2}}{4} \varphi_{i^{\prime}} \varphi_{j^{\prime}}\right) \gamma_{i, i^{\prime}}(\overline{\boldsymbol{\theta}}) \gamma_{j, j^{\prime}}(\overline{\boldsymbol{\theta}})\right] \\
& \quad \times \exp \left(-\frac{\left|\boldsymbol{\theta}-\boldsymbol{\theta}^{\prime}\right|^{2}}{4 \sigma_{W}^{2}}\right)
\end{aligned}
$$

where $\sigma_{\hat{Z}}^{2}$ is defined in Eq. (55). Note that Eq. (57) is very similar to Eq. (45), with the replacement $\sigma_{Z}^{2} \mapsto \sigma_{\hat{Z}}^{2}$. In general $\sigma_{\hat{Z}}^{2}$ is smaller than $\sigma_{Z}^{2}$, since a redshift estimate will hopefully provide a better constraint on the galaxy cosmological weights than using a straight average for all galaxies. We stress, moreover, that $\sigma_{\hat{Z}}^{2}$ is mainly set by the redshift inaccuracies, since it represents the scatter of a measured cosmological weight with respect to the true value, while $\sigma_{Z}^{2}$ depends only on the galaxy redshift distribution. Clearly, if no errors on the redshift estimation are present we have $\sigma_{\hat{Z}}^{2}=0$.

Calculations for the case of a non-vanishing galaxy two-point correlation function, not reported here, are rather surprising. In fact, using the approximations adopted in Sect. 4.3, we obtain a vanishing contribution to the covariance. In other words, the availability of some redshift information completely cancels the noise due to the redshift correlation of background galaxies. In deriving this result, a key role is played by the assumption that the measured cosmological weights are unbiased.

\subsection{Weighted estimator}

The use of an unweighted estimator is rather convenient for the discussion of the noise properties of the shear, but is not optimal. In fact, we can take further advantage of the knowledge of the redshifts of galaxies by suppressing galaxies which are (or are taken to be) in front of the cluster and enhancing distant galaxies, which are well affected by the lens. A simple investigation shows that the weight to use for each galaxy is proportional to $Z^{(n)}$. As a result, we can think to use the shear estimator (see also Lombardi \& Bertin 1999b)

$$
\hat{\gamma}(\boldsymbol{\theta})=\frac{\sum_{n=1}^{N} \epsilon^{(n)} \hat{Z}^{(n)} W\left(\boldsymbol{\theta}-\boldsymbol{\theta}^{(n)}\right)}{\sum_{n=1}^{N}\left(\hat{Z}^{(n)}\right)^{2} W\left(\boldsymbol{\theta}-\boldsymbol{\theta}^{(n)}\right)} .
$$

Unfortunately, this estimator turns out to be biased if the estimated cosmological weights $\hat{Z}^{(n)}$ are not identical to the true ones $Z^{(n)}$. In fact we have

$\langle\hat{\gamma}(\boldsymbol{\theta})\rangle=\frac{\left\langle Z^{2}\right\rangle}{\left\langle\hat{Z}^{2}\right\rangle} \int_{\Omega} W\left(\boldsymbol{\theta}-\boldsymbol{\theta}^{\prime}\right) \gamma\left(\boldsymbol{\theta}^{\prime}\right) \mathrm{d}^{2} \theta$.

In other words, we will underestimate the shear by a factor $1+\sigma_{\hat{Z}}^{2} /\langle Z\rangle^{2}$. This, hopefully, is a number very close to one, so we will for the moment ignore this effect (for example, assuming $\sigma_{\hat{Z}}^{2}<\sigma_{Z}^{2}$, this factor differs from 1 by an amount smaller than 0.02 for a lens at redshift $z_{\mathrm{d}}=0.3$ ).

The expression for the covariance of $\hat{\gamma}$ is in general rather complicated because of the presence of complex combinations of averages of $Z$ and $\hat{Z}$. For this reason, we do not explicitly write here the general expression. In the special case of no errors (so that $\hat{Z}=Z$ ), however, we obtain the simple result

$$
\begin{aligned}
& \operatorname{Cov}_{i j}\left(\hat{\gamma} ; \boldsymbol{\theta}, \boldsymbol{\theta}^{\prime}\right)=\frac{1}{4 \pi\left\langle Z^{2}\right\rangle \rho \sigma_{W}^{2}}\left[\sigma_{\epsilon}^{2} \delta_{i j}\right. \\
& \left.\quad+\left(\frac{\left\langle Z^{2}\right\rangle \sigma_{W}^{2}}{2} \delta_{i^{\prime} j^{\prime}}-\frac{\langle Z\rangle^{2}}{4} \varphi_{i^{\prime}} \varphi_{j^{\prime}}\right) \gamma_{i, i^{\prime}}(\overline{\boldsymbol{\theta}}) \gamma_{j, j^{\prime}}(\overline{\boldsymbol{\theta}})\right] \\
& \quad \times \exp \left(-\frac{\left|\boldsymbol{\theta}-\boldsymbol{\theta}^{\prime}\right|^{2}}{4 \sigma_{W}^{2}}\right)
\end{aligned}
$$

This equation should be compared with Eq. (57) with $\sigma_{\hat{Z}}^{2}=0$. We see that the main difference is the factor $\left\langle Z^{2}\right\rangle$ which replaces $\langle Z\rangle^{2}$. We can thus conclude that, in case of complete redshift knowledge, the estimator (58) performs better than the one of Eq. (56). Actually, the gain is rather low (about $10 \%$ for the lens at redshift $z_{\mathrm{d}}=0.3$ ), and one could also prefer to keep the unweighted estimator (56) rather than the weighted one as long as the lens redshift is much smaller than the median redshift of galaxies.

\subsection{Discussion}

As shown above, one can well take advantage of redshift information to reduce the noise of shear estimates. Since weak lensing analysis normally deals with thousands of galaxies, redshift information is generally available only though photometric redshifts, and thus errors on redshifts must be expected.

In general, galaxy redshifts, even with errors, are very valuable data: they can be used to severely reduce the noise of the mass reconstruction, especially the contribution due to galaxy correlations; moreover, they can also be used to select background galaxies or, more generally, to weight galaxies depending on the lensing signal that they carry. On the other hand, some care should be used when photometric redshifts are used to select galaxies. In fact, 
errors on photometric redshifts are extremely sensitive to the accuracy of the photometry and on the bands used. Large errors, clearly, imply some kind of "contamination" between foreground and background galaxies and, ultimately, introduce a bias on the shear estimate (see above Eq. (59)).

In general, we find that photometric redshifts can almost always be used together with the estimator (56) (which, we recall, can be significantly less noisy than estimators not accounting for galaxy redshifts). We think, instead, that the estimator (58), should be used only if errors on the redshifts are statistically well known and not too large or, alternatively, if the lens is at high redshift. In fact, for a high redshift lens, we expect a significant fraction of foreground galaxies: the use of a "filter," provided by the weight $Z^{(\mathrm{n})}$ in the numerator of Eq. (58), can significantly reduce the noise of the shear and thus of the mass map.

\section{Conclusions}

In this paper we have presented a careful statistical analysis of the shear estimators most widely used in weak lensing mass reconstructions. In particular, we have obtained analytical expressions for the bias and the covariance of the estimators, and identified the various contributions to the noise, as summarized in the following items.

- Intrinsic spread of ellipticities. This term, which is common to all estimators, is basically the only one considered by previous investigations (see Kaiser et al. 1995; Lombardi \& Bertin 1998a; van Waerbeke 2000).

- Poisson noise. Poisson noise is due to the spread of galaxies in positions and redshift. We have shown that a simple, balanced estimator is able to eliminate the angular component of Poisson noise, thus reducing this source of error by a factor $\sim 4$. If redshift information is available, redshift Poisson noise can also be reduced using suitable estimators.

- Correlation noise. The clustering of galaxies introduces a further source of noise. We have shown that this is generally the leading noise contribution, even for the balanced estimator. This source of noise, in contrast to other sources, does not formally decrease with the density of background galaxies. This error can be severely depressed if redshift information is available.

We have also discussed the dependence of these noise terms on several relevant parameters, such as the lens redshift, the lens strength, the density of background galaxies, and the smoothing length used. Finally, we have tested our analytical framework by performing numerical integrations in a specific model, and by comparing the results obtained with our analytical predictions. We have then proved that our finite-form expressions for the shear noise are correct to within 10\%; moreover, we have shown that these expressions can be safely taken also as good approximations for the error on the mass map.

Acknowledgements. We would like to thank Giuseppe Bertin for helpful discussions.

\section{Appendix A: Linear approximation}

For the study of the properties of the estimator (5) we have used a standard technique often applied in statistics (see, e.g., Eadie et al. 1971). Suppose that $X$ is a multidimensional random variable and $Y=f(X)$, with $f$ a smooth function. If $X$ has small covariance, we can expand $f$ to first-order and obtain this way a simple expression for the mean and the covariance of $Y$ :

$\langle Y\rangle \simeq f(\langle X\rangle)$

$\operatorname{Cov}_{i j}(Y) \simeq C_{i i^{\prime}} C_{j j^{\prime}} \operatorname{Cov}_{i^{\prime} j^{\prime}}(X)$,

where

$C_{i j}=\left.\frac{\partial f_{i}}{\partial X_{j}}\right|_{X=\langle X\rangle}$

The goodness of the first-order approximation can be verified by using a second-order expansion. Calculations are particularly simple for the average value:

$\langle Y\rangle \simeq f(\langle X\rangle)+\frac{1}{2} \frac{\partial^{2} f}{\partial X_{1} \partial X_{j}} \operatorname{Cov}_{i j}(X)$.

The second term represents the second-order correction and can be used as an estimate for the error made.

\section{Appendix B: Detailed statistical analysis}

In this Appendix we will carry out in some detail a statistical analysis for the shear estimator (5), thus recovering the results stated in Sect. 5 .

\section{B.1. Average value}

The average value of the estimator is easily evaluated using the linear approximation. In particular, we have

$\langle N\rangle=\rho\langle Z\rangle \int_{\Omega} W\left(\boldsymbol{\theta}-\boldsymbol{\theta}^{\prime}\right) \gamma\left(\boldsymbol{\theta}^{\prime}\right) \mathrm{d}^{2} \theta^{\prime}$,

$\langle D\rangle=\rho\langle Z\rangle$.

As a result, using Eq. (A.1), we find

$\langle\hat{\gamma}(\boldsymbol{\theta})\rangle=\int_{\Omega} W\left(\boldsymbol{\theta}-\boldsymbol{\theta}^{\prime}\right) \gamma\left(\boldsymbol{\theta}^{\prime}\right) \mathrm{d}^{2} \theta^{\prime}$

\section{B.2. Covariance}

Calculations for the covariances are more difficult. The scatter of the numerator from the average value can be written as

$$
\begin{aligned}
N_{i}-\left\langle N_{i}\right\rangle=\sum_{n=1}^{N}[ & \left(\epsilon_{i}^{\mathrm{s}(n)}+\gamma_{i}\left(\boldsymbol{\theta}^{(n)}\right) Z^{(n)}\right) W\left(\boldsymbol{\theta}-\boldsymbol{\theta}^{(n)}\right) \\
& \left.-\frac{\langle Z\rangle}{A} \int_{\Omega} W(\boldsymbol{\theta}-\boldsymbol{\phi}) \gamma_{i}(\boldsymbol{\phi}) \mathrm{d}^{2} \phi\right] .
\end{aligned}
$$


For the denominator we have

$D-\langle D\rangle=\langle Z\rangle \sum_{n=1}^{N}\left[W\left(\boldsymbol{\theta}-\boldsymbol{\theta}^{(n)}\right)-\frac{1}{A}\right]$.

Using Eq. (B.4), we can write the covariance $\operatorname{Cov}\left(N_{i} N_{j}^{\prime}\right)$ as $\operatorname{Cov}\left(N_{i} N_{j}^{\prime} ; \boldsymbol{\theta}, \boldsymbol{\theta}^{\prime}\right)=$

$$
\begin{gathered}
\left\langle\sum _ { n = 1 } ^ { N } \left[\left(\epsilon_{i}^{\mathrm{s}(n)}+\gamma_{i}\left(\boldsymbol{\theta}^{(n)}\right) Z^{(n)}\right) W\left(\boldsymbol{\theta}-\boldsymbol{\theta}^{(n)}\right)\right.\right. \\
\left.-\frac{\langle Z\rangle}{A} \int_{\Omega} W(\boldsymbol{\theta}-\boldsymbol{\phi}) \gamma_{i}(\boldsymbol{\phi}) \mathrm{d}^{2} \phi\right] \times \\
\sum_{m=1}^{N}\left[\left(\epsilon_{j}^{\mathrm{s}(m)}+\gamma_{j}\left(\boldsymbol{\theta}^{(m)}\right) Z^{(m)}\right) W\left(\boldsymbol{\theta}^{\prime}-\boldsymbol{\theta}^{(m)}\right)\right. \\
\left.\left.\quad-\frac{\langle Z\rangle}{A} \int_{\Omega} W\left(\boldsymbol{\theta}^{\prime}-\boldsymbol{\phi}^{\prime}\right) \gamma_{j}\left(\boldsymbol{\phi}^{\prime}\right) \mathrm{d}^{2} \phi^{\prime}\right]\right\rangle .
\end{gathered}
$$

Similarly to Eq. (21), it is convenient here to write $\operatorname{Cov}\left(N_{i} N_{j}^{\prime}\right)=K_{1}+K_{2}$, where $K_{1}$ includes terms for which $n=m$, and $K_{2}$ terms for which $n \neq m$.

If $n=m$, Eq. (8) can be used, leading to

$$
\begin{aligned}
K_{1}= & \rho \sigma_{\epsilon}^{2} \delta_{i j} \int_{\Omega} W(\boldsymbol{\theta}-\boldsymbol{\phi}) W\left(\boldsymbol{\theta}^{\prime}-\boldsymbol{\phi}\right) \mathrm{d}^{2} \phi \\
& +\left\langle Z^{2}\right\rangle \rho \int_{\Omega} \gamma_{i}(\boldsymbol{\phi}) \gamma_{j}(\boldsymbol{\phi}) W(\boldsymbol{\theta}-\boldsymbol{\phi}) W\left(\boldsymbol{\theta}^{\prime}-\boldsymbol{\phi}\right) \mathrm{d}^{2} \phi
\end{aligned}
$$

where terms which vanish in the limit $A \rightarrow \infty$ have been discarted. If $n \neq m$ we have

$$
\begin{aligned}
K_{2}= & \rho^{2} \int_{\Omega} \mathrm{d}^{2} \phi W(\boldsymbol{\theta}-\boldsymbol{\phi}) \gamma_{i}(\boldsymbol{\phi}) \int_{\Omega} \mathrm{d}^{2} \phi^{\prime} W\left(\boldsymbol{\theta}^{\prime}-\boldsymbol{\phi}^{\prime}\right) \gamma_{j}\left(\boldsymbol{\phi}^{\prime}\right) \\
& \times \int_{0}^{\infty} p_{z}(z) Z(z) \mathrm{d} z \int_{0}^{\infty} p_{z}\left(z^{\prime}\right) Z\left(z^{\prime}\right) \xi\left(\boldsymbol{\phi}-\boldsymbol{\phi}^{\prime}, z, z^{\prime}\right) \mathrm{d} z^{\prime} .
\end{aligned}
$$

We recall (see Eq. (25)) that this integral involving the two-point correlation function has been denoted as $\Xi\left[W \gamma_{i} Z W^{\prime} \gamma_{j}^{\prime} Z^{\prime}\right]$. Finally we find for $\operatorname{Cov}\left(N_{i} N_{j}^{\prime}\right)$

$$
\begin{aligned}
\operatorname{Cov} & \left(N_{i} N_{j}^{\prime} ; \boldsymbol{\theta}, \boldsymbol{\theta}^{\prime}\right)=\rho \sigma_{\epsilon}^{2} \delta_{i j} \int_{\Omega} W(\boldsymbol{\theta}-\boldsymbol{\phi}) W\left(\boldsymbol{\theta}^{\prime}-\boldsymbol{\phi}\right) \mathrm{d}^{2} \phi \\
& +\left\langle Z^{2}\right\rangle \rho \int_{\Omega} \gamma_{i}(\boldsymbol{\phi}) \gamma_{j}(\boldsymbol{\phi}) W(\boldsymbol{\theta}-\boldsymbol{\phi}) W\left(\boldsymbol{\theta}^{\prime}-\boldsymbol{\phi}\right) \mathrm{d}^{2} \phi \\
& +\rho^{2} \Xi\left[W \gamma_{i} Z W^{\prime} \gamma_{j}^{\prime} Z^{\prime}\right] .
\end{aligned}
$$

Covariances for the other terms can be calculated in a similar manner. The final result obtained for $\operatorname{Cov}\left(N_{i} D^{\prime}\right)$ is

$$
\begin{aligned}
\operatorname{Cov}\left(N_{i} D^{\prime} ; \boldsymbol{\theta}, \boldsymbol{\theta}^{\prime}\right)= & \langle Z\rangle^{2} \rho \int_{\Omega} W(\boldsymbol{\theta}-\boldsymbol{\phi}) W\left(\boldsymbol{\theta}^{\prime}-\boldsymbol{\phi}\right) \gamma_{i}(\boldsymbol{\phi}) \mathrm{d}^{2} \phi \\
& +\langle Z\rangle \rho^{2} \Xi\left[W \gamma_{i} Z W^{\prime}\right] .
\end{aligned}
$$

The result for $\operatorname{Cov}\left(D N_{j}^{\prime}\right)$ is analogous and will not be written explicitely. Finally, the last covariance needed is

$$
\begin{aligned}
\operatorname{Cov}\left(D D^{\prime} ; \boldsymbol{\theta}, \boldsymbol{\theta}^{\prime}\right)= & \langle Z\rangle^{2} \rho \int_{\Omega} W(\boldsymbol{\theta}-\boldsymbol{\phi}) W\left(\boldsymbol{\theta}^{\prime}-\boldsymbol{\phi}\right) \mathrm{d}^{2} \phi \\
& +\langle Z\rangle^{2} \rho^{2} \Xi\left[W W^{\prime}\right] .
\end{aligned}
$$

In principle, we could now evaluate the covariance of $\hat{\gamma}$ using the results obtained so far. In practice, the resulting expression is by far too complex to be of any practical use at this stage. We thus avoid to write this expression in the general case, but rather we will consider the slowly varying shear approximation in the next Appendix.

\section{Appendix C: Slowly varying shear}

We will derive here the slowly varying shear expressions for the results obtained in Appendix B. Again, only the estimator (5) will be considered; calculations for the estimator (4) are very similar.

\section{C.1. Average value}

To first order, the average value of $\hat{\gamma}$ is just the ratio between $\langle N\rangle$ and $\langle D\rangle$ (see Eq. (A.1)). Let us focus on $\langle N\rangle$, which is given by Eq. (B.1). Inserting Eqs. (32) in (B.1) and using the symmetry of $W$ we get

$$
\langle N\rangle=\rho\langle Z\rangle \gamma(\boldsymbol{\theta}) \int_{\Omega} W\left(\boldsymbol{\theta}-\boldsymbol{\theta}^{\prime}\right) \mathrm{d}^{2} \boldsymbol{\theta}^{\prime}=\rho\langle Z\rangle \gamma(\boldsymbol{\theta}) .
$$

Finally, using Eq. (A.1) we obtain

$\langle\hat{\gamma}(\boldsymbol{\theta})\rangle=\gamma(\boldsymbol{\theta})$.

\section{C.2. Covariance $(\xi=0)$}

Calculations for the covariance can be similarly carried out using an expansion for $\gamma$ around the mid-point $\overline{\boldsymbol{\theta}}=$ $\left(\boldsymbol{\theta}+\boldsymbol{\theta}^{\prime}\right) / 2$ (see Fig. 1). This is convenient because with this choice several linear terms containing partial derivatives of $\gamma$ vanish. Hence we have

$$
\begin{aligned}
\operatorname{Cov}_{i j}\left(\hat{\gamma} ; \boldsymbol{\theta}, \boldsymbol{\theta}^{\prime}\right)=\frac{1}{\rho}\left[\frac{\sigma_{\epsilon}^{2}}{\langle Z\rangle^{2}} \delta_{i j}+\frac{\left\langle Z^{2}\right\rangle}{\langle Z\rangle^{2}} \gamma_{i}(\overline{\boldsymbol{\theta}}) \gamma_{j}(\overline{\boldsymbol{\theta}})\right. \\
\left.\quad-\gamma_{i}(\boldsymbol{\theta}) \gamma_{j}(\overline{\boldsymbol{\theta}})-\gamma_{i}(\overline{\boldsymbol{\theta}}) \gamma_{j}\left(\boldsymbol{\theta}^{\prime}\right)+\gamma_{i}(\boldsymbol{\theta}) \gamma_{j}\left(\boldsymbol{\theta}^{\prime}\right)\right] \\
\times(W \star W)\left(\boldsymbol{\theta}-\boldsymbol{\theta}^{\prime}\right) \\
+\frac{\left\langle Z^{2}\right\rangle}{\langle Z\rangle^{2} \rho} \gamma_{i, i^{\prime}}(\overline{\boldsymbol{\theta}}) \gamma_{j, j^{\prime}}(\overline{\boldsymbol{\theta}}) \\
\quad \times \int_{\Omega} W(\boldsymbol{\theta}-\boldsymbol{\phi}) W\left(\boldsymbol{\theta}^{\prime}-\boldsymbol{\phi}\right)\left(\phi_{i^{\prime}}-\bar{\theta}_{i^{\prime}}\right)\left(\phi_{j^{\prime}}-\bar{\theta}_{j^{\prime}}\right) \mathrm{d}^{2} \phi
\end{aligned}
$$

We can apply once more the slowly varying shear approximation to this result. In the case where $W$ is a Gaussian the final result can be written in a simpler form:

$$
\begin{aligned}
& \operatorname{Cov}_{i j}\left(\hat{\gamma} ; \boldsymbol{\theta}, \boldsymbol{\theta}^{\prime}\right)=\frac{1}{4 \pi\langle Z\rangle^{2} \rho \sigma_{W}^{2}}\left[\sigma_{\epsilon}^{2} \delta_{i j}+\sigma_{Z}^{2} \gamma_{i}(\overline{\boldsymbol{\theta}}) \gamma_{j}(\overline{\boldsymbol{\theta}})\right. \\
& \left.\quad+\left(\frac{\left\langle Z^{2}\right\rangle \sigma_{W}^{2}}{2} \delta_{i^{\prime} j^{\prime}}-\frac{\langle Z\rangle^{2}}{4} \varphi_{i^{\prime}} \varphi_{j^{\prime}}\right) \gamma_{i, i^{\prime}}(\overline{\boldsymbol{\theta}}) \gamma_{j, j^{\prime}}(\overline{\boldsymbol{\theta}})\right] \\
& \quad \times \exp \left(-\frac{\left|\boldsymbol{\theta}-\boldsymbol{\theta}^{\prime}\right|^{2}}{4 \sigma_{W}^{2}}\right)
\end{aligned}
$$




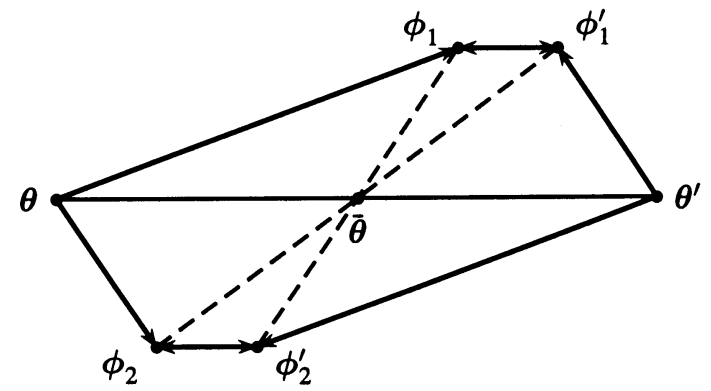

Fig. C.1. Similarly to Fig. 1, this graph shows that linear terms of the expansion of $\gamma$ around $\overline{\boldsymbol{\theta}}$ do not contribute to the term $C_{2}$. In fact, the integrand evaluated at the upper configuration $\left(\phi_{1}, \phi_{1}^{\prime}\right)$ is the opposite of the integrand evaluated at lower configuration $\left(\phi_{2}, \phi_{2}^{\prime}\right)$.

\section{C.3. Check of the linear approximation}

We can explicitely verify the goodness of the first-order approximation by checking the second-order terms of Eq. (A.4). These terms are both of the form

$$
\frac{\gamma(\boldsymbol{\theta})}{\rho}\left[\int_{\Omega} W\left(\boldsymbol{\theta}^{\prime}\right) W\left(\boldsymbol{\theta}^{\prime}\right) \mathrm{d}^{2} \theta^{\prime}\right]=\frac{\gamma(\boldsymbol{\theta})}{\rho A_{W}} .
$$

The two terms have opposite sign. In other words, the average value of $\hat{\gamma}$ obtained above is correct to second order [actually it is easy to show that the estimator (5) really is unbiased to all orders]. We also note that the relative contribution of this terms is of the order $1 /\left(\rho A_{W}\right) \sim 1 / N_{W}$, where, we recall, $N_{W}$ the expected number of galaxies inside the effective area of $W$. Note that $1 / N_{W}$ is also the expected relative variance of the denominator $D$ of Eq. (5). We have thus recovered the condition needed to perform the linear approximation, already discussed at the end of Sect. 5.1.

\section{C.4. Covariance $(\xi \neq 0)$}

Given the difficulties encountered to study the contribution to the covariance from the two-point correlation function, we will carry out the calculations only in the case where $W$ is a Gaussian of the form (3).

Let us focus on a specific term involving the twopoint correlation function, for example the last term of Eq. (B.9), i.e. $\Xi_{i j} \equiv \Xi\left[W \gamma_{i} Z W^{\prime} \gamma_{j}^{\prime} Z^{\prime}\right]$ [in this section, for simplicity, we will use the symbols $\Xi_{i j}, \Xi_{i}$, and $\Xi$ to denote, respectively, the last terms of Eqs. (B.9), (B.10), and (B.11)]. We will use the expression (12) for the function $\xi$ with the approximate exponent $\eta=2$. We then write

$\xi\left(\boldsymbol{\delta}, z, z^{\prime}\right)=\frac{d_{0}^{2}(1+z)^{\alpha}}{D_{\mathrm{A}}^{2}(z)|\boldsymbol{\delta}|^{2}+\left(D_{\mathrm{P}}(z)-D_{\mathrm{P}}\left(z^{\prime}\right)\right)^{2}}$,

where $\boldsymbol{\delta}=\boldsymbol{\phi}-\boldsymbol{\phi}^{\prime}$ must be expressed in radians. In this equation $D_{\mathrm{A}}(z)$ and $D_{\mathrm{P}}(z)$ are, respectively, the angular diameter distance and the proper distance of an object at redshift $z$. The length-scale of $\xi$ is set by $d_{0}$, and typically is a few Mpc. As a result, the two-point correlation function vanishes unless $z^{\prime}$ is very close to $z$ (this property will be repeatedly used below). On the other hand, all other functions involving redshifts, namely $D_{\mathrm{A}}, D_{\mathrm{P}}$ (but also $p_{z}$ and $Z$ ), are slowly varying with $z$. We thus can approximate $\xi$ as

$\xi\left(\boldsymbol{\delta}, z, z^{\prime}\right) \simeq \frac{d_{0}^{2}(1+z)^{\alpha}}{D_{\mathrm{A}}^{2}(z)|\boldsymbol{\delta}|^{2}+\left[D_{\mathrm{P}}^{\prime}(z)\right]^{2}\left(z^{\prime}-z\right)^{2}}$.

Moreover, since $\xi$ is basically zero for $z^{\prime} \neq z$, we can take as constant the term $p_{z}\left(z^{\prime}\right) Z\left(z^{\prime}\right)$ in Eq. (23), and perform directly the integration over $z^{\prime}$. We get then

$$
\begin{aligned}
\Xi_{i j} \simeq & \int_{\Omega} \mathrm{d}^{2} \phi W(\boldsymbol{\theta}-\boldsymbol{\phi}) \gamma_{i}(\boldsymbol{\phi}) \int_{\Omega} \mathrm{d}^{2} \phi^{\prime} W\left(\boldsymbol{\theta}^{\prime}-\boldsymbol{\phi}^{\prime}\right) \gamma_{j}\left(\boldsymbol{\phi}^{\prime}\right) \\
& \times \int_{0}^{\infty}\left[p_{z}(z) Z(z)\right]^{2} \mathrm{~d} z \int_{0}^{\infty} \xi\left(\boldsymbol{\phi}-\boldsymbol{\phi}^{\prime}, z, z^{\prime}\right) \mathrm{d} z^{\prime} .
\end{aligned}
$$

Using Eq. (C.7) the last integration is trivial:

$\int_{0}^{\infty} \xi\left(\boldsymbol{\delta}, z, z^{\prime}\right) \mathrm{d} z^{\prime}=\frac{d_{0}^{2}(1+z)^{\alpha}}{D_{\mathrm{A}}(z) D_{\mathrm{P}}^{\prime}(z)|\boldsymbol{\delta}|}[\arctan t]_{t_{0}}^{\infty}$

with

$t_{0}=-\frac{D_{\mathrm{P}}^{\prime}(z) z}{D_{\mathrm{A}}(z)|\boldsymbol{\delta}|}$

In normal conditions $t_{0}$ has always a very large negative value (unless $z$ is exceedingly small; these cases, however, are of no interest in weak lensing studies). We thus can safely replace $t_{0}$ with $-\infty$ in Eq. (C.9), thus obtaining

$\int_{0}^{\infty} \xi\left(\boldsymbol{\delta}, z, z^{\prime}\right) \mathrm{d} z^{\prime} \simeq \frac{\pi d_{0}^{2}(1+z)^{\alpha}}{D_{\mathrm{A}}(z) D_{\mathrm{P}}^{\prime}(z)|\boldsymbol{\delta}|} \equiv \zeta(\boldsymbol{\delta}, z)$.

We now can perform the two integrals on $\boldsymbol{\theta}$ and $\boldsymbol{\theta}^{\prime}$. Since the results obtained so far depends only on $\delta=\phi^{\prime}-\phi$, we have to evaluate two "quasi-convolutions," the "quasi" being due to the fact that the integrand also contain terms like $\gamma_{i}(\phi)$ and $\gamma_{j}\left(\phi^{\prime}\right)$. Hence we can apply the slowly varying field approximation (see Fig. C.1), obtaining the integral

$$
\begin{aligned}
\Xi_{i j} \simeq & \gamma_{i}(\overline{\boldsymbol{\theta}}) \gamma_{j}(\overline{\boldsymbol{\theta}}) \int_{0}^{\infty}\left[p_{z}(z)\right]^{2}[Z(z)]^{2} \mathrm{~d} z \int_{\Omega} W(\boldsymbol{\theta}-\boldsymbol{\phi}) \mathrm{d}^{2} \phi \\
& \times \int_{\Omega} W\left(\boldsymbol{\theta}^{\prime}-\boldsymbol{\phi}^{\prime}\right) \zeta\left(\boldsymbol{\phi}^{\prime}-\boldsymbol{\phi}, z\right) \mathrm{d}^{2} \phi .
\end{aligned}
$$

Using the properties of convolutions we can recast the two integrals on the angular variables in the form

$\nu(\boldsymbol{\varphi}, z)=\int_{\Omega} \zeta(\phi, z)(W \star W)(\boldsymbol{\varphi}-\boldsymbol{\phi}) \mathrm{d}^{2} \boldsymbol{\phi}$,

where $\boldsymbol{\varphi}=\boldsymbol{\theta}^{\prime}-\boldsymbol{\theta}$. Using the Gaussian weight function (3) and the result obtained above for $\zeta$, we can perform the integration in polar coordinates. The result is

$\nu(\boldsymbol{\varphi}, z)=\frac{\pi^{3 / 2} d_{0}^{2}(1+z)^{\alpha}}{2 D_{\mathrm{A}}(z) D_{\mathrm{P}}^{\prime}(z) \sigma_{W}} I_{0}\left(\frac{|\boldsymbol{\varphi}|^{2}}{8 \sigma_{W}^{2}}\right) \exp \left(-\frac{|\boldsymbol{\varphi}|^{2}}{8 \sigma_{W}^{2}}\right)$, 


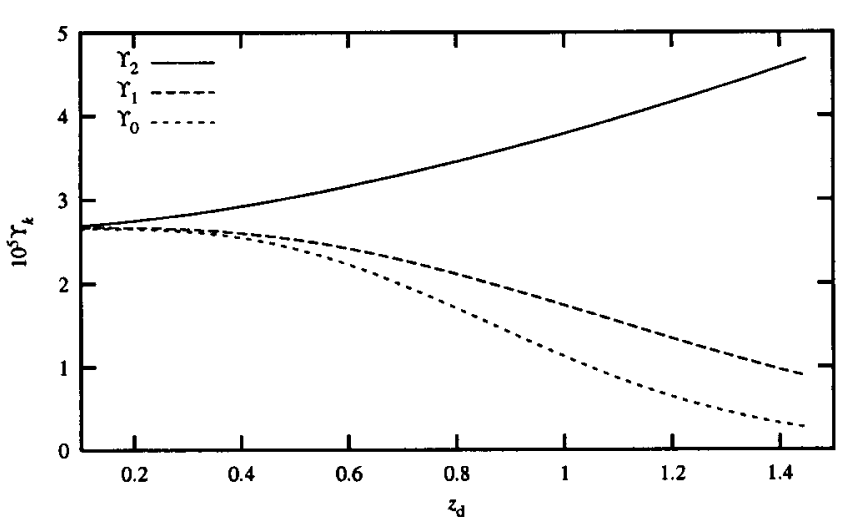

Fig. C.2. The dimensionless quantities $\Upsilon_{k}$ as a function of the lens redshift $z_{\mathrm{d}}$.

where $I_{0}$ is the modified Bessel function of first kind. The final result for the covariance term is thus

$$
\begin{aligned}
\Xi_{i j}= & \frac{\pi^{3 / 2} d_{0}^{2}}{2 \sigma_{W}} \gamma_{i}(\overline{\boldsymbol{\theta}}) \gamma_{j}(\overline{\boldsymbol{\theta}}) \eta\left(\frac{|\boldsymbol{\varphi}|^{2}}{8 \sigma_{W}^{2}}\right) \\
& \times \int_{z_{d}}^{\infty} \frac{(1+z)^{\alpha}\left[p_{z}(z) Z(z)\right]^{2}}{D_{\mathrm{A}}(z) D_{\mathrm{P}}^{\prime}(z)} \mathrm{d} z .
\end{aligned}
$$

The function $\eta(x)=I_{0}(x) \exp (-x)$ represents the leading angular dependence. The last integral cannot be recast in a simple form and must be evaluated numerically (it also depends on the cosmological model). We stress that, since $\boldsymbol{\delta}$ in Eq. (C.6) has been taken to be expressed in radians, we similarly need to express $\sigma_{W}$ in radians in Eq. (C.15).

Calculations for the other integrals involving the twopoint correlation function, namely $\Xi_{i}$ and $\Xi$, are very similar. Defining the dimensionless quantities

$\Upsilon_{k}=\frac{\pi^{3 / 2} d_{0}^{2}}{2\langle Z\rangle^{k}} \int_{z_{d}}^{\infty} \frac{(1+z)^{\alpha}\left[p_{z}(z)\right]^{2}[Z(z)]^{k}}{D_{\mathrm{A}}(z) D_{\mathrm{P}}^{\prime}(z)} \mathrm{d} z$,

the various two-point correlation terms can be written as

$$
\begin{aligned}
\Xi_{i j} & =\frac{\langle Z\rangle^{2}}{\sigma_{W}} \gamma_{i}(\overline{\boldsymbol{\theta}}) \gamma_{j}(\overline{\boldsymbol{\theta}}) \eta\left(\frac{|\boldsymbol{\varphi}|^{2}}{8 \sigma_{W}^{2}}\right) \Upsilon_{2}, \\
\Xi_{i} & =\frac{\langle Z\rangle}{\sigma_{W}} \gamma_{i}(\overline{\boldsymbol{\theta}}) \eta\left(\frac{|\boldsymbol{\varphi}|^{2}}{8 \sigma_{W}^{2}}\right) \Upsilon_{1}, \\
\Xi & =\frac{1}{\sigma_{W}} \eta\left(\frac{|\boldsymbol{\varphi}|^{2}}{8 \sigma_{W}^{2}}\right) \Upsilon_{0} .
\end{aligned}
$$

In summary, the contribution to the $\operatorname{Cov}(\hat{\gamma})$ from a nonvanishing two-point correlation function is

$$
\begin{aligned}
C_{2}=\frac{1}{\sigma_{W}}[ & \Upsilon_{2} \gamma_{i}(\overline{\boldsymbol{\theta}}) \gamma_{j}(\overline{\boldsymbol{\theta}})+\Upsilon_{0} \gamma_{i}(\boldsymbol{\theta}) \gamma_{j}\left(\boldsymbol{\theta}^{\prime}\right)-\Upsilon_{1} \gamma_{i}(\boldsymbol{\theta}) \gamma_{j}(\overline{\boldsymbol{\theta}}) \\
& \left.-\Upsilon_{1} \gamma_{i}(\overline{\boldsymbol{\theta}}) \gamma_{j}\left(\boldsymbol{\theta}^{\prime}\right)\right] \eta\left(\frac{\left|\boldsymbol{\theta}-\boldsymbol{\theta}^{\prime}\right|^{2}}{8 \sigma_{W}^{2}}\right) \cdot \quad \text { (C.20) }
\end{aligned}
$$

Note that $\eta(x) \propto 1 / \sqrt{x}$ for large $x$, and thus this contribution to the covariance is the leading term at large separations $|\phi|$.

In the case $\boldsymbol{\theta}=\boldsymbol{\theta}^{\prime}$ this expression can be written in the more compact form

$C_{2}=\frac{\Upsilon_{2}+\Upsilon_{0}-2 \Upsilon_{1}}{\sigma_{W}} \gamma_{i}(\boldsymbol{\theta}) \gamma_{j}(\boldsymbol{\theta})$.
For a lens at redshift $z_{\mathrm{d}}=0.3$ we have $\Upsilon_{2} \simeq 2.8267 \times 10^{-5}$, $\Upsilon_{1} \simeq 2.6513 \times 10^{-5}$, and $\Upsilon_{0} \simeq 2.6246 \times 10^{-5}$, so that $\Upsilon_{2}+\Upsilon_{0}-2 \Upsilon_{1} \simeq 1.488 \times 10^{-6}$. A plot of the quantities $\Upsilon_{k}$ as a function of the lens redshift is provided in Fig. C.2.

For Eq. (4) we obtain similar results, but now only the term $\Xi_{i j}$ contributes to the covariance. More specifically, we find

$C_{2}=\frac{\Upsilon_{2}}{\sigma_{W}} \gamma_{i}(\overline{\boldsymbol{\theta}}) \gamma_{j}(\overline{\boldsymbol{\theta}}) \eta\left(\frac{\left|\boldsymbol{\theta}-\boldsymbol{\theta}^{\prime}\right|^{2}}{8 \sigma_{W}^{2}}\right)$

As a result, we get a much larger error, since the effect of cancellation of terms clearly shown by Eq. (C.21) is no longer present. In particular, for the typical case considered here, we expect a correlation about 19 times larger, i.e. an error about 4 times larger.

\section{Appendix D: Calculations for estimators with source redshifts}

In this appendix we briefly sketch the calculations leading to the results described in Sect. 8 .

\section{D.1. Unweighted estimator}

Let us call $N$ and $D$ the numerator and the denominator of the r.h.s. of Eq. (56), respectively. Assuming unbiased measured cosmological weights, we easily find

$$
\begin{aligned}
& \langle N\rangle=\rho\langle Z\rangle \int_{\Omega} W\left(\boldsymbol{\theta}-\boldsymbol{\theta}^{\prime}\right) \gamma\left(\boldsymbol{\theta}^{\prime}\right) \mathrm{d}^{2} \theta^{\prime}, \\
& \langle D\rangle=\rho\langle Z\rangle
\end{aligned}
$$

so that Eq. (56) is recovered.

Regarding the covariance, we find after some calculations

$$
\begin{aligned}
\operatorname{Cov} & \left(N_{i} D^{\prime} ; \boldsymbol{\theta}, \boldsymbol{\theta}^{\prime}\right)= \\
& \left\langle Z^{2}\right\rangle \rho \int_{\Omega} W(\boldsymbol{\theta}-\boldsymbol{\phi}) W\left(\boldsymbol{\theta}^{\prime}-\boldsymbol{\phi}\right) \gamma_{i}(\boldsymbol{\phi}) \mathrm{d}^{2} \phi \\
& +\rho^{2} \Xi\left[W \gamma_{i} Z W^{\prime} Z^{\prime}\right] \\
\operatorname{Cov}( & \left.D D^{\prime} ; \boldsymbol{\theta}, \boldsymbol{\theta}^{\prime}\right)=\left\langle\hat{Z}^{2}\right\rangle \rho \int_{\Omega} W(\boldsymbol{\theta}-\boldsymbol{\phi}) W\left(\boldsymbol{\theta}^{\prime}-\boldsymbol{\phi}\right) \mathrm{d}^{2} \phi \\
& +\rho^{2} \Xi\left[W Z W^{\prime} Z^{\prime}\right]
\end{aligned}
$$

Note that, since the numerator is formally identical to the numerator of Eq. (5), Eq. (B.9) still holds. Differences with respect to the analogous terms calculated above in Eqs. (B.10) and (B.11) are the presence of $\left\langle Z^{2}\right\rangle$ and $\left\langle\hat{Z}^{2}\right\rangle$ factors instead of $\langle Z\rangle^{2}$ and the different form of the correlation terms, which now include always the redshift $Z$. Substituting these expressions in Eq. (44) and using the slow varying shear approximation we obtain Eq. (57). 


\section{D.2. Weighted estimator}

The slowly varying shear approximation applied to the numerator $N$ and denominator $D$ of Eq. (58) gives

$$
\begin{aligned}
\langle N\rangle & =\rho\langle Z\rangle \int_{\Omega} W\left(\boldsymbol{\theta}-\boldsymbol{\theta}^{\prime}\right) \gamma\left(\boldsymbol{\theta}^{\prime}\right) \mathrm{d}^{2} \theta^{\prime} \\
\langle D\rangle & =\rho\langle Z\rangle .
\end{aligned}
$$

These equations lead immediately to Eq. (59).

Regarding the covariances, we find

$$
\begin{aligned}
\operatorname{Cov} & \left(N_{i} N_{j}^{\prime} ; \boldsymbol{\theta}, \boldsymbol{\theta}^{\prime}\right)=\sigma_{\epsilon}^{2}\left\langle\hat{Z}^{2}\right\rangle \delta_{i j} \int_{\Omega} W\left(\boldsymbol{\theta}-\boldsymbol{\theta}^{\prime \prime}\right) W\left(\boldsymbol{\theta}^{\prime}-\boldsymbol{\theta}^{\prime \prime}\right) \mathrm{d}^{2} \theta^{\prime \prime} \\
& +\left\langle Z^{2} \hat{Z}^{2}\right\rangle \rho \int_{\Omega} \gamma_{i}\left(\boldsymbol{\theta}^{\prime \prime}\right) \gamma_{j}\left(\boldsymbol{\theta}^{\prime \prime}\right) W\left(\boldsymbol{\theta}-\boldsymbol{\theta}^{\prime \prime}\right) W\left(\boldsymbol{\theta}^{\prime}-\boldsymbol{\theta}^{\prime \prime}\right) \mathrm{d}^{2} \theta^{\prime \prime} \\
& +\rho^{2} \Xi\left[W^{\prime \prime} \gamma_{i}^{\prime \prime} Z^{\prime \prime} \hat{Z}^{\prime \prime} W^{\prime \prime \prime} \gamma_{j}^{\prime \prime \prime} Z^{\prime \prime \prime} \hat{Z}^{\prime \prime \prime}\right],
\end{aligned}
$$

$\operatorname{Cov}\left(N_{i} D^{\prime} ; \boldsymbol{\theta}, \boldsymbol{\theta}^{\prime}\right)=$

$$
\begin{aligned}
& +\left\langle Z \hat{Z}^{3}\right\rangle \rho \int_{\Omega} W\left(\boldsymbol{\theta}-\boldsymbol{\theta}^{\prime \prime}\right) W\left(\boldsymbol{\theta}^{\prime}-\boldsymbol{\theta}^{\prime \prime}\right) \gamma_{i}\left(\boldsymbol{\theta}^{\prime \prime}\right) \mathrm{d}^{2} \theta^{\prime \prime} \\
& +\rho^{2} \Xi\left[W^{\prime \prime} \gamma_{i}^{\prime \prime} Z^{\prime \prime} \hat{Z}^{\prime \prime}\left(\hat{Z}^{\prime \prime \prime}\right)^{2} W^{\prime \prime \prime}\right],
\end{aligned}
$$

$\operatorname{Cov}\left(D D^{\prime} ; \boldsymbol{\theta}, \boldsymbol{\theta}^{\prime}\right)=\left\langle\hat{Z}^{4}\right\rangle \rho \int_{\Omega} W\left(\boldsymbol{\theta}-\boldsymbol{\theta}^{\prime \prime}\right) W\left(\boldsymbol{\theta}^{\prime}-\boldsymbol{\theta}^{\prime \prime}\right) \mathrm{d}^{2} \theta^{\prime \prime}$

$$
+\rho^{2} \Xi\left[W^{\prime \prime}\left(\hat{Z}^{\prime \prime}\right)^{2} W^{\prime \prime \prime}\left(\hat{Z}^{\prime \prime \prime}\right)^{2}\right] .
$$

These expressions can be used to derive Eq. (60).

\section{References}

Bartelmann, M., Narayan, R., Seitz, S., \& Schneider, P. 1996, ApJ, 464, L115

Bartelmann, M., \& Schneider, P. 2001, Phys. Rep., 340, 291

Benitez, N. 2000, ApJ, 536, 571

Bertin, G., \& Lombardi, M. 2001, ApJ, 546, 47

Brainerd, T. G., Blandford, R. D., \& Smail, I. 1996, ApJ, 466, 623

Clowe, D., Luppino, G., Kaiser, N., Henry, J., \& Gioia, I. 1998, ApJ, 497, L61

Eadie, W., Drijard, D., James, F., Roos, M., \& Sadoulet, B. 1971, Statistical Methods in Experimental Physics (Amsterdam New-York Oxford: North-Holland Publishing Company)
Fahlman, G., Kaiser, N., Squires, G., \& Woods, D. 1994, ApJ, 437, 56

Fischer, P. 1999, AJ, 117, 2024

Fischer, P., Bernstein, G., Rhee, G., \& Tyson, J. A. 1997, AJ, 113,521

Fischer, P., \& Tyson, J. A. 1997, AJ, 114, 14

Hoekstra, H., Franx, M., Kuijken, K., \& Squires, G. 1998, ApJ, 504,636

Kaiser, N. 1995, ApJ, 439, L1

Kaiser, N., \& Squires, G. 1993, ApJ, 404, 441

Kaiser, N., Squires, G., \& Broadhurst, T. 1995, ApJ, 449, 460

Kochanek, C. S. 1990, MNRAS, 247, 135

Le Fèvre, O., Hudon, D., Lilly, S. J., et al. 1996, ApJ, 461, 534

Lombardi, M., \& Bertin, G. 1998a, A\&A, 335, 1

Lombardi, M., \& Bertin, G. 1998b, A\&A, 330, 791

Lombardi, M., \& Bertin, G. 1999a, A\&A, 348, 38

Lombardi, M., \& Bertin, G. 1999b, A\&A, 342, 337

Lombardi, M., Rosati, P., Nonino, M., et al. 2000, A\&A, 363, 401

Lombardi, M., \& Schneider, P. 2001, A\&A, 373, 359

Luppino, G. A., \& Kaiser, N. 1997, ApJ, 475, 20

Mellier, Y. 1999, ARA\&A, 37, 127

Peebles, P. J. E. 1993, Princiles of Physical Cosmology (Princeton: Princeton University Press)

Schneider, P. 1995, A\&A, 302, 639

Schneider, P., \& Seitz, C. 1995, A\&A, 294, 411

Seitz, C., Kneib, J.-P., Schneider, P., \& Seitz, S. 1996, A\&A, 314,707

Seitz, C., \& Schneider, P. 1995, A\&A, 297, 287

Seitz, C., \& Schneider, P. 1997, A\&A, 318, 687

Seitz, S., \& Schneider, P. 1996, A\&A, 305, 383

Seitz, S., \& Schneider, P. 2001, A\&A, 374, 740

Seitz, S., Schneider, P., \& Bartelmann, M. 1998, A\&A, 337, 325

Smail, I., Couch, W., Ellis, R., \& Sharples, R. 1995, ApJ, 440, 501

Squires, G., \& Kaiser, N. 1996, ApJ, 473, 65

Squires, G., Kaiser, N., Babul, A., et al. 1996, ApJ, 461, 572

Squires, G., Kaiser, N., Fahlman, G., Babul, A., \& Woods, D. 1996, ApJ, 469, 73

Tyson, J., Wenk, R., \& Valdes, F. 1990, ApJ, 349, L1

Tyson, J. A., \& Fischer, P. 1995, ApJ, 446, L55

van Waerbeke, L. 2000, MNRAS, 313, 524

Webster, R. 1985, MNRAS, 213, 871 